\title{
The first Pontryagin class of a quadratic Lie 2-algebroid
}

\author{
Yunhe Sheng \\ Department of Mathematics, Jilin University, Changchun 130012, China \\ Email: shengyh@jlu.edu.cn
}

\begin{abstract}
In this paper, first we give a detailed study on the structure of a transitive Lie 2-algebroid and describe a transitive Lie 2-algebroid using a morphism from the tangent Lie algebroid $T M$ to a strict Lie 3-algebroid constructed from derivations. Then we introduce the notion of a quadratic Lie 2-algebroid and define its first Pontryagin class, which is a cohomology class in $H^{5}(M)$. Associated to a CLWX 2-algebroid, there is a quadratic Lie 2-algebroid naturally. Conversely, we show that the first Pontryagin class of a quadratic Lie 2-algebroid is the obstruction class of the existence of a CLWX-extension. Finally we construct a quadratic Lie 2-algebroid from a trivial principle 2-bundle with a $\Gamma$-connection and show that its first Pontryagin class is trivial.
\end{abstract}

\section{Contents}

1 Introduction 2

2 Preliminaries 3

2.1 Leibniz 2-algebras . . . . . . . . . . . . . . . . . . . . . . . . . . . . . . . . . . . .

2.2 Lie $n$-algebroids and CLWX 2-algebroids . . . . . . . . . . . . . . 5

3 Transitive Lie 2-algebroids 6

3.1 General description of a transitive Lie 2-algebroid . . . . . . . . . . . . . . . 6

3.2 Transitive Lie 2-algebroids and Lie 3 -algebroid morphisms . . . . . . . . . . . . 8

4 The first Pontryagin class of a quadratic Lie 2-algebroid 11

5 Exact CLWX 2-algebroids 15

5.1 Skeletal exact CLWX 2-algebroids . . . . . . . . . . . . . . . . 16

5.2 CLWX-extension of a quadratic Lie 2-algebroid . . . . . . . . . . . . . . 17

6 The first Pontryagin class of a trivial principle 2-bundle with a $\Gamma$-connection 21

6.1 Strict Lie 2 -groups and strict Lie 2 -algebras . . . . . . . . . . . . . . . . . . . 21

6.2 The transitive Lie 2-algebroid associated to a trivial principle 2-bundle with a $\Gamma$ -

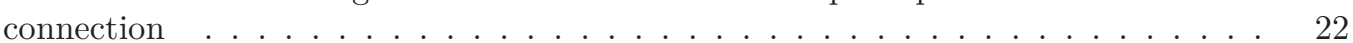

6.3 The first Pontryagin class of the quadratic Lie 2-algebroid associated to a $\Gamma$-connection 24

\footnotetext{
${ }^{0}$ Key words: transitive Lie 2-algebroid, quadratic Lie 2-algebroid, the first Pontryagin class, CLWX 2-algebroid, principle 2-bundle, $\Gamma$-connection

${ }^{0} M S C: 17 \mathrm{~B} 99,53 \mathrm{D} 17$.
} 


\section{Introduction}

The notion of a Lie algebroid was introduced by Pradines in 1967, which is a generalization of Lie algebras and tangent bundles. Just as Lie algebras are the infinitesimal objects of Lie groups, Lie algebroids are the infinitesimal objects of Lie groupoids. See 16] for general theory about Lie algebroids. A transitive Lie algebroid (i.e. $\rho$ is surjective) $\left(A,[\cdot, \cdot]_{A}, \rho\right)$ is called a quadratic Lie algebroid if there is a nondegenerate symmetric invariant bilinear form $\mathcal{S}$ on $\operatorname{ker}(\rho)$. A connection of a quadratic Lie algebroid is a splitting $\sigma: T M \longrightarrow A$ of the short exact sequence

$$
0 \longrightarrow \operatorname{ker}(\rho) \longrightarrow A \stackrel{\rho}{\longrightarrow} T M \longrightarrow 0 .
$$

The curvature $R \in \operatorname{Hom}\left(\wedge^{2} T M, \operatorname{ker}(\rho)\right)$ is defined by

$$
R(X, Y)=\sigma[X, Y]-[\sigma(X), \sigma(Y)]_{A}, \quad \forall X, Y \in \Gamma(T M) .
$$

Then the first Pontryagin class of a quadratic Lie algebroid is defined to be the cohomology class $[\mathcal{S}(R, R)]$ in $H^{4}(M)$.

The notion of a Courant algebroid was introduced in [14] in the study of the double of a Lie bialgebroid. An alternative definition was given in [20]. See the review article [12] for more information. Roughly speaking, a Courant algebroid is a vector bundle $E$, whose section space is a Leibniz algebra, together with an anchor map $\rho: E \longrightarrow T M$ and a nondegenerate symmetric bilinear form $S$, such that some compatibility conditions are satisfied. For a transitive Courant algebroid (i.e. $\rho$ is surjective), $E / \rho^{*}\left(T^{*} M\right)$ is a quadratic Lie algebroid. Conversely, it is proved in [8, 9] that a quadratic Lie algebroid admits a Courant-extension if and only if its first Pontryagin class is trivial.

Recently, people have paid more attention to higher categorical structures by reasons in both mathematics and physics. A Lie 2-algebra is the categorification of a Lie algebra [3]. The Jacobi identity is replaced by a natural transformation, called the Jacobiator, which also satisfies some coherence laws of its own. If a skew-symmetric bracket is used in the definition of a Courant algebroid, the authors showed that the underlying algebraic structure of a Courant algebroid is a Lie 2-algebra [21]. Along this approach, the author defined split Lie $n$-algebroids in [26] using the language of graded vector bundles. Usually an NQ-manifold of degree $n$ is considered as a Lie $n$-algebroid [27. The equivalence between the category of split Lie $n$-algebroids and the category of NQ-manifolds of degree $n$ was given in [6].

CLWX 2-algebroids (named after Courant-Liu-Weinstein-Xu) were introduced in [13], which can be viewed as the categorification of Courant algebroids, and one-to-one correspond to symplectic NQ-manifolds of degree 3. See 11 for more applications in 4D topological field theory. Similar as the case of Courant algebroids, we can obtain a quadratic Lie 2-algebroid (called the ample Lie 2-algebroid) from an exact CLWX 2-algebroid by modulo $\rho^{*}\left(T^{*} M\right)$. Then it is natural to ask the following question:

- Whether every quadratic Lie 2-algebroid admits a CLWX-extension? If not, what is the obstruction?

Motivated by the above question, first we study the structure of a transitive Lie 2-algebroid $\left(A_{-1} \oplus A_{0} ; \rho, l_{1}, l_{2}, l_{3}\right)$. It turns out that a transitive Lie 2 -algebroid is a nonabelian extension of the tangent Lie algebroid $T M$ by a graded bundle of Lie 2 -algebra $A_{-1} \oplus \operatorname{ker}(\rho)$. By choosing a splitting (a connection), we give precise formulas of a transitive Lie 2-algebroid. One important property that we need to stress here is that the corresponding curvatures include a $\operatorname{ker}(\rho)$-valued 2 -form 
and an $A_{-1}$-valued 3-form. Furthermore, we construct a strict Lie 3-algebroid using derivations of $A_{-1} \oplus \operatorname{ker}(\rho)$ and characterize a transitive Lie 2-algebroid by a morphism from the tangent Lie algebroid $T M$ to this strict Lie 3-algebroid. Then we go on to give the notion of a quadratic Lie 2-algebroid. For a quadratic Lie 2-algebroid, we construct a 5-form using the aforementioned curvatures. We show that this 5 -form is closed and does not depend on the choices of connections. Thus, it gives rise to a characteristic class, which we call the first Pontryagin class of a quadratic Lie 2-algebroid. The first Pontryagin class of a quadratic Lie 2-algebroid plays the same role as the one for a quadratic Lie algebroid, namely, a quadratic Lie 2-algebroid admits a CLWX-extension if and only if its first Pontryagin class is trivial. This is the reason why we call this characteristic class the first Pontryagin class of a quadratic Lie 2-algebroid.

In the past ten years, principle 2-bundles and higher gauge theory are deeply studied in 2, 5, 17, 10, 17, 18, 22, 223, 28, 29, 30. For a trivial principle $\Gamma$-2-bundle with a connection, where $\Gamma$ is a strict Lie 2-group, we construct a transitive Lie 2-algebroid which can be viewed as its Atiyah algebroid. We further show that if the Lie 2-algebra corresponding to the Lie 2 -group $\Gamma$ is quadratic, then this Lie 2-algebroid is quadratic and its first Pontryagin class is trivial. We will go on to study the infinitesimal of principle 2-bundles in a separate paper.

The paper is organized as follows. In Section 2, we recall Leibniz 2-algebras, Lie $n$-algebroids and CLWX 2-algebroids. In Section 3, we study transitive Lie 2-algebroids. In Subsection 3.1, we give precise conditions that the structure maps of a transitive Lie 2-algebroid satisfy. In Subsection 3.2 , we construct a strict Lie 3-algebroid using derivations and characterize a transitive Lie 2algebroid by a morphism from the tangent Lie algebroid $T M$ to this strict Lie 3-algebroid. In Section 4, we give the notion of a quadratic Lie 2-algebroid and define its first Pontryagin class, which is a cohomology class in $H^{5}(M)$. In Section 5, we study exact CLWX 2-algebroids. In Subsection 5.1, we show that skeletal exact CLWX 2-algebroids are classified by the higher analogue of the Ševera class, which is a cohomology class in $H^{4}(M)$. In Subsection 5.2, we show that a CLWX 2-algebroid gives rise to a quadratic Lie 2-algebroid and conversely, a quadratic Lie 2algebroid admits a CLWX-extension if and only if its first Pontryagin class is trivial. In Section 6, for a trivial principle 2-bundle with a $\Gamma$-connection, we construct a transitive Lie 2-algebroid and show that its first Pontryagin class is trivial.

Acknowledgement: We give our warmest thanks to Zhangju Liu, Konrad Waldorf, Xiaomeng $\mathrm{Xu}$ and Chenchang Zhu for very useful comments and discussions. We also give our special thanks to the referee for very helpful suggestions that improve the paper. This research is supported by NSFC (11471139) and NSF of Jilin Province (20170101050JC).

\section{Preliminaries}

\subsection{Leibniz 2-algebras}

As a model for "Leibniz algebras that satisfy Jacobi identity up to all higher homotopies", the notion of a strongly homotopy Leibniz algebra, or a $\operatorname{Lod}_{\infty}$-algebra was given in 15 by Livernet, which was further studied by Ammar and Poncin in [1. In [25], the authors introduced the notion of a Leibniz 2-algebra, which is the categorification of a Leibniz algebra, and proved that the category of Leibniz 2-algebras and the category of 2-term $\operatorname{Lod}_{\infty}$-algebras are equivalent.

Definition 2.1. A Leibniz 2-algebra $\mathcal{V}$ consists of the following data:

- a complex of vector spaces $\mathcal{V}: V_{-1} \stackrel{\mathrm{d}}{\longrightarrow} V_{0}$, 
- bilinear maps $l_{2}: V_{-i} \times V_{-j} \longrightarrow V_{-i-j}$, where $0 \leq i+j \leq 1$,

- a trilinear map $l_{3}: V_{0} \times V_{0} \times V_{0} \longrightarrow V_{-1}$,

such that for all $w, x, y, z \in V_{0}$ and $m, n \in V_{-1}$, the following equalities are satisfied:

(a) $\mathrm{d} l_{2}(x, m)=l_{2}(x, \mathrm{~d} m)$,

(b) $\mathrm{d}_{2}(m, x)=l_{2}(\mathrm{~d} m, x)$,

(c) $l_{2}(\mathrm{~d} m, n)=l_{2}(m, \mathrm{~d} n)$,

(d) $\mathrm{d} l_{3}(x, y, z)=l_{2}\left(x, l_{2}(y, z)\right)-l_{2}\left(l_{2}(x, y), z\right)-l_{2}\left(y, l_{2}(x, z)\right)$,

$\left(\mathrm{e}_{1}\right) l_{3}(x, y, \mathrm{~d} m)=l_{2}\left(x, l_{2}(y, m)\right)-l_{2}\left(l_{2}(x, y), m\right)-l_{2}\left(y, l_{2}(x, m)\right)$,

$\left(\mathrm{e}_{2}\right) l_{3}(x, \mathrm{~d} m, y)=l_{2}\left(x, l_{2}(m, y)\right)-l_{2}\left(l_{2}(x, m), y\right)-l_{2}\left(m, l_{2}(x, y)\right)$,

$\left(\mathrm{e}_{3}\right) l_{3}(\mathrm{~d} m, x, y)=l_{2}\left(m, l_{2}(x, y)\right)-l_{2}\left(l_{2}(m, x), y\right)-l_{2}\left(x, l_{2}(m, y)\right)$,

(f) the Jacobiator identity:

$$
\begin{aligned}
& l_{2}\left(w, l_{3}(x, y, z)\right)-l_{2}\left(x, l_{3}(w, y, z)\right)+l_{2}\left(y, l_{3}(w, x, z)\right)+l_{2}\left(l_{3}(w, x, y), z\right) \\
& -l_{3}\left(l_{2}(w, x), y, z\right)-l_{3}\left(x, l_{2}(w, y), z\right)-l_{3}\left(x, y, l_{2}(w, z)\right) \\
& +l_{3}\left(w, l_{2}(x, y), z\right)+l_{3}\left(w, y, l_{2}(x, z)\right)-l_{3}\left(w, x, l_{2}(y, z)\right)=0 .
\end{aligned}
$$

We usually denote a Leibniz 2-algebra by $\left(V_{-1} \oplus V_{0} ; \mathrm{d}, l_{2}, l_{3}\right)$, or simply by $\mathcal{V}$. In particular, if both $l_{2}$ and $l_{3}$ are skew-symmetric, we obtain the notion of a Lie 2 -algebra [3].

Definition 2.2. Let $\mathcal{V}$ and $\mathcal{V}^{\prime}$ be Leibniz 2-algebras, a morphism $\mathfrak{f}$ from $\mathcal{V}$ to $\mathcal{V}^{\prime}$ consists of

- linear maps $f_{0}: V_{0} \longrightarrow V_{0}^{\prime}$ and $f_{1}: V_{1} \longrightarrow V_{1}^{\prime}$ commuting with the differential, i.e.

$$
f_{0} \circ \mathrm{d}=\mathrm{d}^{\prime} \circ f_{1}
$$

- a bilinear map $f_{2}: V_{0} \times V_{0} \longrightarrow V_{1}^{\prime}$,

such that for all $x, y, z \in L_{0}, m \in L_{1}$, we have

$$
\left\{\begin{aligned}
f_{0} l_{2}(x, y)-l_{2}^{\prime}\left(f_{0}(x), f_{0}(y)\right) & =\mathrm{d}^{\prime} f_{2}(x, y), \\
f_{1} l_{2}(x, m)-l_{2}^{\prime}\left(f_{0}(x), f_{1}(m)\right) & =f_{2}(x, \mathrm{~d} m), \\
f_{1} l_{2}(m, x)-l_{2}^{\prime}\left(f_{1}(m), f_{0}(x)\right) & =f_{2}(\mathrm{~d} m, x),
\end{aligned}\right.
$$

and

$$
\begin{aligned}
& -f_{1}\left(l_{3}(x, y, z)\right)+l_{2}^{\prime}\left(f_{0}(x), f_{2}(y, z)\right)-l_{2}^{\prime}\left(f_{0}(y), f_{2}(x, z)\right)-l_{2}^{\prime}\left(f_{2}(x, y), f_{0}(z)\right) \\
& -f_{2}\left(l_{2}(x, y), z\right)+f_{2}\left(x, l_{2}(y, z)\right)-f_{2}\left(y, l_{2}(x, z)\right)+l_{3}^{\prime}\left(f_{0}(x), f_{0}(y), f_{0}(z)\right)=0 .
\end{aligned}
$$




\subsection{Lie $n$-algebroids and CLWX 2-algebroids}

The notion of a split Lie $n$-algebroid was introduced in [26].

Definition 2.3. $A$ split Lie $n$-algebroid is a graded vector bundle $\mathcal{A}=A_{-n+1} \oplus \cdots \oplus A_{-1} \oplus A_{0}$ over a manifold $M$ equipped with a bundle map (the anchor) $\rho: A_{0} \longrightarrow T M$, and brackets $l_{i}$ : $\Gamma\left(\wedge^{i} \mathcal{A}\right) \longrightarrow \Gamma(\mathcal{A})$ with degree $2-i$ for $i=1,2, \cdots, l_{n+1}$, such that

(1) $\left(\Gamma(\mathcal{A}) ; l_{1}, l_{2}, \cdots, l_{n+1}\right)$ is a Lie $n$-algebra (n-term $L_{\infty}$-algebra);

(2) $l_{2}$ satisfies the Leibniz rule with respect to the anchor $\rho$ :

$$
l_{2}\left(X^{0}, f Y\right)=f l_{2}\left(X^{0}, Y\right)+\rho\left(X^{0}\right)(f) Y, \quad \forall X^{0} \in \Gamma\left(A_{0}\right), f \in C^{\infty}(M), Y \in \Gamma(\mathcal{A}) ;
$$

(3) for $i \neq 2, l_{i}$ are $C^{\infty}(M)$-linear.

Denote a Lie $n$-algebroid by $\left(\mathcal{A} ; \rho, l_{1}, l_{2}, \cdots, l_{n+1}\right)$.

A split Lie $n$-algebroid is said to be strict if $l_{i}=0$ for all $i>2$. In this paper, we will only use split Lie 2-algebroids and strict split Lie 3-algebroids. See 6] for more details about the category of Lie $n$-algebroids.

Lemma 2.4. Let $\left(\mathcal{A} ; \rho, l_{1}, l_{2}, \cdots, l_{n+1}\right)$ be a Lie $n$-algebroid. Then we have

$$
\begin{aligned}
\rho \circ l_{1} & =0, \\
\rho l_{2}\left(X^{0}, Y^{0}\right) & =\left[\rho\left(X^{0}\right), \rho\left(Y^{0}\right)\right], \quad \forall X^{0}, Y^{0} \in \Gamma\left(A_{0}\right) .
\end{aligned}
$$

When $n=1$, a Lie 1-algebroid is exactly a Lie algebroid. Associated to any vector bundle $E$, the covariant differential operator bundle $\mathfrak{D}(E)$ is a Lie algebroid naturally. Let $\left(A ; a, l_{2}\right)$ be a Lie algebroid and $\nabla: A \longrightarrow \mathfrak{D}(E)$ a bundle map. Then there is a differential operator $d_{\nabla}: \Gamma\left(\operatorname{Hom}\left(\wedge^{k} A, E\right)\right) \longrightarrow \Gamma\left(\operatorname{Hom}\left(\wedge^{k+1} A, E\right)\right)$ defined by

$$
\begin{aligned}
d_{\nabla} \theta\left(X_{1}, \cdots, X_{k+1}\right)= & \sum_{i=1}^{k+1}(-1)^{i+1} \nabla_{X_{i}} \theta\left(X_{1}, \cdots, \widehat{X}_{i}, \cdots, X_{k+1}\right) \\
& +\sum_{i<j}(-1)^{i+j} \theta\left(l_{2}\left(X_{i}, X_{j}\right), X_{1}, \cdots, \widehat{X_{i}}, \cdots, \widehat{X_{j}}, \cdots, X_{k+1}\right),
\end{aligned}
$$

for all $X_{1}, \cdots, X_{k+1} \in \Gamma(A) . \quad d_{\nabla}^{2}=0$ if and only if $\nabla$ is a Lie algebroid morphism, i.e. a representation of $A$ on $E$. See [16] for more details.

The notion of a CLWX 2-algebroid was introduced in [13] as the categorification of a Courant algebroid [14, 20].

Definition 2.5. A CLWX 2-algebroid is a graded vector bundle $\mathcal{E}=E_{-1} \oplus E_{0}$ over $M$ equipped with a nondegenerate symmetric bilinear form $S$ on $\mathcal{E}$, a bilinear operation $\diamond: \Gamma\left(E_{-i}\right) \times \Gamma\left(E_{-j}\right) \longrightarrow$ $\Gamma\left(E_{-(i+j)}\right), 0 \leq i+j \leq 1$, which is skewsymmetric restricted on $\Gamma\left(E_{0}\right) \times \Gamma\left(E_{0}\right)$, an $E_{-1}$-valued 3 -form $\Omega$ on $E_{0}$, two bundle maps $\partial: E_{-1} \longrightarrow E_{0}$ and $\rho: E_{0} \longrightarrow T M$, such that $E_{-1}$ and $E_{0}$ are isotropic and the following conditions are satisfied:

(i) $\left(\Gamma\left(E_{-1}\right), \Gamma\left(E_{0}\right), \partial, \diamond, \Omega\right)$ is a Leibniz 2-algebra,

(ii) for all $e \in \Gamma(\mathcal{E})$, $e \diamond e=\frac{1}{2} \mathcal{D} S(e, e)$, 
(iii) for all $e_{1}^{1}, e_{2}^{1} \in \Gamma\left(E_{-1}\right), S\left(\partial\left(e_{1}^{1}\right), e_{2}^{1}\right)=S\left(e_{1}^{1}, \partial\left(e_{2}^{1}\right)\right)$,

(iv) for all $e_{1}, e_{2}, e_{3} \in \Gamma(\mathcal{E}), \rho\left(e_{1}\right) S\left(e_{2}, e_{3}\right)=S\left(e_{1} \diamond e_{2}, e_{3}\right)+S\left(e_{2}, e_{1} \diamond e_{3}\right)$,

(v) for all $e_{1}^{0}, e_{2}^{0}, e_{3}^{0}, e_{4}^{0} \in \Gamma\left(E_{0}\right), S\left(\Omega\left(e_{1}^{0}, e_{2}^{0}, e_{3}^{0}\right), e_{4}^{0}\right)=-S\left(e_{3}^{0}, \Omega\left(e_{1}^{0}, e_{2}^{0}, e_{4}^{0}\right)\right)$,

where $\mathcal{D}: C^{\infty}(M) \longrightarrow \Gamma\left(E_{-1}\right)$ is defined by

$$
S\left(\mathcal{D} f, e^{0}\right)=\rho\left(e^{0}\right)(f), \quad \forall f \in C^{\infty}(M), e^{0} \in \Gamma\left(E_{0}\right) .
$$

Denote a CLWX 2-algebroid by $\left(E_{-1}, E_{0}, \partial, \rho, S, \diamond, \Omega\right)$, or simply by $\mathcal{E}$. The skew-symmetrization of the Leibniz 2-algebra structure $\diamond$ will give rise to a Lie 3 -algebra structure. There is a close relationship between CLWX 2-algebroids and symplectic NQ manifolds (QP manifolds) of degree 3. On the double of a Lie 2-bialgebroid, there is a CLWX 2-algebroid structure naturally. See [11, 13] for more details.

\section{Transitive Lie 2-algebroids}

In this section, we study the structure of a transitive Lie 2-algebroid. In Subsection 3.1, by choosing a splitting, we obtain some structure maps and we give the conditions that these structure maps satisfy. Then in Subsection 3.2, we characterize these conditions by a morphism from the tangent Lie algebroid $T M$ to a strict Lie 3-algebroid constructed from derivations of a graded bundle of Lie 2-algebras.

\subsection{General description of a transitive Lie 2-algebroid}

A Lie 2-algebroid $\left(A_{-1} \oplus A_{0} ; \rho, l_{1}, l_{2}, l_{3}\right)$ is said to be transitive if the anchor $\rho: A_{0} \longrightarrow T M$ is surjective. Denote by $\mathcal{G}=\operatorname{ker}(\rho)$. By Lemma 2.4, we deduce that $l_{2}(u, v) \in \Gamma(\mathcal{G})$ for all $u, v \in \Gamma(\mathcal{G})$. Then it is obvious that $\left(A_{-1} \oplus \mathcal{G} ; \mathfrak{l}_{1}, \mathfrak{l}_{2}, \mathfrak{l}_{3}\right)$ is a graded bundle of Lie 2 -algebras, where $\mathfrak{l}_{1}=l_{1}, \mathfrak{l}_{2}$ and $\mathfrak{l}_{3}$ are restrictions of $l_{2}$ and $l_{3}$ respectively.

Definition 3.1. A splitting (connection) of a transitive Lie 2-algebroid $\left(A_{-1} \oplus A_{0} ; \rho, l_{1}, l_{2}, l_{3}\right)$ consists of a section $\sigma: T M \longrightarrow A_{0}$ of the following short exact sequence of vector bundles:

$$
0 \longrightarrow \mathcal{G} \stackrel{\text { i }}{\longrightarrow} A_{0} \stackrel{\rho}{\longrightarrow} T M \longrightarrow 0,
$$

and a bundle map $\gamma: \wedge^{2} T M \longrightarrow A_{-1}$. Here i denotes the inclusion map.

After choosing a splitting, we have $A_{0} \cong T M \oplus \mathcal{G}$ and $\rho$ is simply the projection $\operatorname{pr}_{T M}$. Define $\nabla^{0}: T M \longrightarrow \mathfrak{D}(\mathcal{G})$ and $\nabla^{1}: T M \longrightarrow \mathfrak{D}\left(A_{-1}\right)$ by

$$
\nabla_{X}^{0} u=l_{2}(\sigma(X), u), \quad \nabla_{X}^{1} m=l_{2}(\sigma(X), m), \quad \forall X \in \mathfrak{X}(M), u \in \Gamma(\mathcal{G}), m \in \Gamma\left(A_{-1}\right) .
$$

Define the bundle map $R: \wedge^{2} T M \longrightarrow \mathcal{G}$ by

$$
R(X, Y)=\sigma[X, Y]-l_{2}(\sigma(X), \sigma(Y))-\mathfrak{l}_{1} \gamma(X, Y), \quad \forall X, Y \in \mathfrak{X}(M) .
$$

Define the bundle map $I: \wedge^{3} T M \longrightarrow A_{-1}$ by

$$
I(X, Y, Z)=-d_{\nabla^{1}} \gamma(X, Y, Z)-l_{3}(\sigma(X), \sigma(Y), \sigma(Z)), \quad \forall X, Y, Z \in \mathfrak{X}(M) .
$$


Define totally skewsymmetric bundle map $J: \wedge^{2} T M \otimes \mathcal{G} \longrightarrow A_{-1}$ by

$$
J(X, Y, u)=-l_{3}(\sigma(X), \sigma(Y), u), \quad \forall X, Y \in \mathfrak{X}(M), \quad u \in \Gamma(\mathcal{G}) .
$$

Define totally skewsymmetric bundle map $K: T M \otimes \wedge^{2} \mathcal{G} \longrightarrow A_{-1}$ by

$$
K(X, u, v)=l_{3}(\sigma(X), u, v), \quad \forall X \in \mathfrak{X}(M), \quad u, v \in \Gamma(\mathcal{G}) .
$$

Transfer the transitive Lie 2-algebroid structure from $A_{-1} \oplus A_{0}$ to $A_{-1} \oplus(\mathcal{G} \oplus T M)$, for which we use the same notation $\left(\rho, l_{1}, l_{2}, l_{3}\right)$, we have

$$
\left\{\begin{aligned}
\rho= & \operatorname{pr}_{T M}, \\
l_{1}(m)= & \mathfrak{l}_{1}(m), \\
l_{2}(X+u, Y+v)= & {[X, Y]-R_{\gamma}(X, Y)+\nabla_{X}^{0} v-\nabla_{Y}^{0} u+\mathfrak{l}_{2}(u, v), } \\
l_{2}(X+u, m)= & -l_{2}(m, X+u)=\nabla_{X}^{1} m+\mathfrak{l}_{2}(u, m), \\
l_{3}(X+u, Y+v, Z+w)= & -I_{\gamma}(X, Y, Z)-J(X, Y, w)-J(X, v, Z)-J(u, Y, Z) \\
& +K(X, v, w)+K(u, Y, w)+K(u, v, Z)+\mathfrak{l}_{3}(u, v, w),
\end{aligned}\right.
$$

where

$$
R_{\gamma}=R+\mathfrak{l}_{1} \circ \gamma, \quad I_{\gamma}=I+d_{\nabla^{1}} \gamma .
$$

Remark 3.2. $R_{\gamma}$ and $I_{\gamma}$ can be viewed as curvatures.

Theorem 3.3. Let $\left(A_{-1} \oplus \mathcal{G} ; \mathfrak{l}_{1}, \mathfrak{l}_{2}, \mathfrak{l}_{3}\right)$ be a graded bundle of Lie 2-algebras. Then $\left(A_{-1} \oplus(\mathcal{G} \oplus\right.$ $\left.T M) ; \rho, l_{1}, l_{2}, l_{3}\right)$ is a transitive Lie 2-algebroid, where $\rho, l_{1}, l_{2}, l_{3}$ are given by (12) for totally skewsymmetric $I, J, K$, if and only if for all $X, Y, Z \in \mathfrak{X}(M), u, v, w \in \Gamma(\mathcal{G})$ and $m \in \Gamma\left(A_{-1}\right)$, the following equalities hold:

$$
\begin{aligned}
\mathfrak{l}_{1} \circ \nabla_{X}^{1} & =\nabla_{X}^{0} \circ \mathfrak{l}_{1}, \\
\nabla_{X}^{0} \mathfrak{l}_{2}(v, w)-\mathfrak{l}_{2}\left(\nabla_{X}^{0} v, w\right)-\mathfrak{l}_{2}\left(v, \nabla_{X}^{0} w\right) & =\mathfrak{l}_{1} K(X, v, w), \\
\nabla_{X}^{1} \mathfrak{l}_{2}(v, m)-\mathfrak{l}_{2}\left(\nabla_{X}^{0} v, m\right)-\mathfrak{l}_{2}\left(v, \nabla_{X}^{1} m\right) & =K\left(X, v, \mathfrak{l}_{1} m\right), \\
\mathfrak{l}_{2}(u, K(v, w, X))-\mathfrak{l}_{2}(v, K(u, w, X))+\mathfrak{l}_{2}(w, K(u, v, X)) & \\
-K\left(\mathfrak{l}_{2}(u, v), w, X\right)-K\left(v, \mathfrak{l}_{2}(u, w), X\right)+K\left(u, \mathfrak{l}_{2}(v, w), X\right) & \\
-\nabla_{X}^{1} \mathfrak{l}_{3}(u, v, w)+\mathfrak{l}_{3}\left(\nabla_{X}^{0} u, v, w\right)+\mathfrak{l}_{3}\left(u, \nabla_{X}^{0} v, w\right)+\mathfrak{l}_{3}\left(u, v, \nabla_{X}^{0} w\right) & =0, \\
\nabla_{X}^{0} \nabla_{Y}^{0} w-\nabla_{Y}^{0} \nabla_{X}^{0} w-\nabla_{[X, Y]}^{0} w+\mathfrak{l}_{2}\left(R_{\gamma}(X, Y), w\right)+\mathfrak{l}_{1} J(X, Y, w) & =0, \\
\nabla_{X}^{1} \nabla_{Y}^{1} m-\nabla_{Y}^{1} \nabla_{X}^{1} m-\nabla_{[X, Y]}^{1} m+\mathfrak{l}_{2}\left(R_{\gamma}(X, Y), m\right)+J\left(X, Y, \mathfrak{l}_{1} m\right) & =0, \\
d_{\nabla^{0}} R_{\gamma}(X, Y, Z) & =\mathfrak{l}_{1} I_{\gamma}(X, Y, Z), \\
-\nabla_{Z}^{1} J(u, X, Y)+J\left(\nabla_{X}^{0} u, Y, Z\right)+J\left(X, \nabla_{Y}^{0} u, Z\right)+J\left(X, Y, \nabla_{Z}^{0} u\right) & \\
+J(u,[X, Y], Z)+J(u,[Z, X], Y)+J(u,[Y, Z], X) & \\
-K\left(u, Z, R_{\gamma}(X, Y)\right)-K\left(u, Y, R_{\gamma}(Z, X)\right)-K\left(u, X, R_{\gamma}(Y, Z)\right) & =0, \\
d_{\nabla^{1}} I_{\gamma}+J \circ R_{\gamma} & =0,
\end{aligned}
$$

where $J \circ R_{\gamma}: \wedge^{4} \mathfrak{X}(M) \longrightarrow \Gamma\left(A_{-1}\right)$ is given by

$$
J \circ R_{\gamma}\left(X_{1}, \cdots, X_{4}\right)=\frac{1}{4} \sum_{\tau \in S_{4}} \operatorname{sgn}(\tau) J\left(R_{\gamma}\left(X_{\tau(1)}, X_{\tau(2)}\right), X_{\tau(3)}, X_{\tau(4)}\right) .
$$


Proof. Assume that $\left(A_{-1} \oplus(\mathcal{G} \oplus T M) ; \rho, l_{1}, l_{2}, l_{3}\right)$ is a transitive Lie 2-algebroid. By the equality $l_{1} l_{2}(X, m)=l_{2}\left(X, l_{1}(m)\right)$, we deduce that (13) holds. By the equality

$$
l_{2}\left(X, l_{2}(v, w)\right)+l_{2}\left(v, l_{2}(w, X)\right)+l_{2}\left(w, l_{2}(X, v)\right)=l_{1} l_{3}(X, v, w),
$$

we deduce that (14) holds. Similarly, we deduce that (15) holds. By the equality

$$
\begin{array}{r}
l_{2}\left(u, l_{3}(v, w, X)\right)-l_{2}\left(v, l_{3}(u, w, X)\right)+l_{2}\left(w, l_{3}(u, v, X)\right)-l_{2}\left(X, l_{3}(u, v, w)\right)-l_{3}\left(l_{2}(u, v), w, X\right) \\
+l_{3}\left(l_{2}(u, w), v, X\right)-l_{3}\left(l_{2}(v, w), u, X\right)-l_{3}\left(l_{2}(u, X), v, w\right)+l_{3}\left(l_{2}(v, X), u, w\right)-l_{3}\left(l_{2}(w, X), u, v\right)=0,
\end{array}
$$

we deduce that (16) holds. By the equality

$$
l_{2}\left(X, l_{2}(Y, w)\right)+l_{2}\left(Y, l_{2}(w, X)\right)+l_{2}\left(w, l_{2}(X, Y)\right)=l_{1} l_{3}(X, Y, w),
$$

we deduce that (17) holds. Similarly, we deduce that (18) holds. By the equality

$$
l_{2}\left(X, l_{2}(Y, Z)\right)+l_{2}\left(Y, l_{2}(Z, X)\right)+l_{2}\left(Z, l_{2}(X, Y)\right)=l_{1} l_{3}(X, Y, Z),
$$

we deduce that (19) holds. Then by the equality

$$
\begin{array}{r}
l_{2}\left(u, l_{3}(X, Y, Z)\right)-l_{2}\left(X, l_{3}(u, Y, Z)\right)+l_{2}\left(Y, l_{3}(u, X, Z)\right)-l_{2}\left(Z, l_{3}(u, X, Y)\right)-l_{3}\left(l_{2}(u, X), Y, Z\right) \\
+l_{3}\left(l_{2}(u, Y), X, Z\right)-l_{3}\left(l_{2}(u, Z), X, Y\right)-l_{3}\left(l_{2}(X, Y), u, Z\right)+l_{3}\left(l_{2}(X, Z), u, Y\right)-l_{3}\left(l_{2}(Y, Z), u, X\right)=0,
\end{array}
$$

we deduce that (20) holds. Finally, by the equality

$$
\sum_{i=1}^{4}(-1)^{i+1} l_{2}\left(X_{i}, l_{3}\left(X_{1}, \cdots, \widehat{X_{i}}, \cdots, X_{4}\right)\right)+\sum_{i<j}(-1)^{i+j} l_{3}\left(l_{2}\left(X_{i}, X_{j}\right), X_{1}, \cdots, \widehat{X_{i}}, \cdots, \widehat{X_{j}}, \cdots, X_{4}\right)=0,
$$

we deduce that (21) holds.

Conversely, by (14) and (17), we can deduce that

$$
\begin{aligned}
-\mathfrak{l}_{2}(u, J(v, Y, Z))+\mathfrak{l}_{2}(v, J(u, Y, Z))+J\left(\mathfrak{l}_{2}(u, v), Y, Z\right) & \\
+\nabla_{Y}^{1} K(u, v, Z)-\nabla_{Z}^{1} K(u, v, Y)-K(u, v,[Y, Z])+\mathfrak{l}_{3}\left(u, v, R_{\gamma}(Y, Z)\right) & \\
+K\left(v, \nabla_{Y}^{0} u, Z\right)+K\left(v, Y, \nabla_{Z}^{0} u\right)-K\left(u, \nabla_{Y}^{0} v, Z\right)-K\left(u, Y, \nabla_{Z}^{0} v\right) & =0 .
\end{aligned}
$$

Then by (13)-(21) and (23), we can deduce that $\left(A_{-1} \oplus(\mathcal{G} \oplus T M) ; \rho, l_{1}, l_{2}, l_{3}\right)$ is a transitive Lie 2-algebroid.

\subsection{Transitive Lie 2-algebroids and Lie 3-algebroid morphisms}

In this subsection, we give a conceptual explanation of equations listed in Theorem 3.3 We show that they give rise to a morphism from the Lie algebroid $T M$ to a strict Lie 3 -algebroid constructed from derivations of the graded bundle of Lie 2-algebras $\left(A_{-1} \oplus \mathcal{G} ; \mathfrak{l}_{1}, \mathfrak{l}_{2}, \mathfrak{l}_{3}\right)$.

First we give the definition of a morphism from a Lie algebroid to a strict Lie 3-algebroid. See [6. Section 4.1] for details for general formulas of a morphism between Lie $n$-algebroids.

Definition 3.4. Let $\mathcal{A}=\left(A ; \rho, l_{2}\right)$ be a Lie algebroid and $\mathcal{A}^{\prime}=\left(A_{-2}^{\prime} \oplus A_{-1}^{\prime} \oplus A_{0}^{\prime} ; \rho^{\prime}, l_{1}^{\prime}, l_{2}^{\prime}\right)$ a strict Lie 3-algebroid. A morphism $F$ from $\mathcal{A}$ to $\mathcal{A}^{\prime}$ consists of:

- a bundle map $F^{1}: A \longrightarrow A_{0}^{\prime}$,

- a bundle map $F^{2}: \wedge^{2} A_{0} \longrightarrow A_{-1}^{\prime}$,

- a bundle map $F^{3}: \wedge^{3} A_{0} \longrightarrow A_{-2}^{\prime}$, 
such that for all $X, Y, Z, X_{i} \in \Gamma(A), i=1,2,3,4$, we have

$$
\begin{aligned}
\rho^{\prime} \circ F^{1} & =\rho, \\
F^{1} l_{2}(X, Y)-l_{2}^{\prime}\left(F^{1}(X), F^{1}(Y)\right) & =l_{1}^{\prime} F^{2}(X, Y), \\
l_{2}^{\prime}\left(F^{1}(X), F^{2}(Y, Z)\right)-F^{2}\left(l_{2}(X, Y), Z\right)+c . p . & =l_{1}^{\prime} F^{3}(X, Y, Z),
\end{aligned}
$$

and

$$
\begin{aligned}
& \sum_{i=1}^{4}(-1)^{i+1} l_{2}^{\prime}\left(F^{1}\left(X_{i}\right), F^{3}\left(X_{1}, \cdots, \widehat{X}_{i}, \cdots X_{4}\right)\right) \\
& +\sum_{i<j}(-1)^{i+j}\left(F^{3}\left(l_{2}\left(X_{i}, X_{j}\right), X_{k}, X_{l}\right)-\frac{1}{2} l_{2}^{\prime}\left(F^{2}\left(X_{i}, X_{j}\right), F^{2}\left(X_{k}, X_{l}\right)\right)\right)=0,
\end{aligned}
$$

where $k<l$ and $\{k, l\} \cap\{i, j\}=\emptyset$.

Then we construct a strict Lie 3-algebroid using derivations of a graded bundle of Lie 2-algebras $\mathfrak{G}=\left(\mathcal{G}_{-1} \oplus \mathcal{G}_{0} ; \mathfrak{l}_{1}, \mathfrak{l}_{2}, \mathfrak{l}_{3}\right)$. Denote by $\mathfrak{D}\left(\mathcal{G}_{0}\right)$ and $\mathfrak{D}\left(\mathcal{G}_{-1}\right)$ the covariant differential operator bundle associated to $\mathcal{G}_{0}$ and $\mathcal{G}_{-1}$ respectively. We have the following exact sequences:

$$
\begin{array}{r}
0 \longrightarrow \mathfrak{g l}\left(\mathcal{G}_{0}\right) \longrightarrow \mathfrak{D}\left(\mathcal{G}_{0}\right) \stackrel{\text { jo }}{\longrightarrow} T M \longrightarrow 0, \\
0 \longrightarrow \mathfrak{g l}\left(\mathcal{G}_{-1}\right) \longrightarrow \mathfrak{D}\left(\mathcal{G}_{-1}\right) \stackrel{\mathfrak{j}_{1}}{\longrightarrow} T M \longrightarrow 0 .
\end{array}
$$

Definition 3.5. A degree 0 derivation of a graded bundle of Lie 2-algebras $\left(\mathcal{G}_{-1} \oplus \mathcal{G}_{0} ; \mathfrak{l}_{1}, \mathfrak{l}_{2}, \mathfrak{l}_{3}\right)$ is a triple $\mathfrak{d}=\left(\mathfrak{d}_{0}, \mathfrak{d}_{1}, l_{\mathfrak{d}}\right)$, where $\mathfrak{d}_{0} \in \Gamma\left(\mathfrak{D}\left(\mathcal{G}_{0}\right)\right), \mathfrak{d}_{1} \in \Gamma\left(\mathfrak{D}\left(\mathcal{G}_{-1}\right)\right)$ and $l_{\mathfrak{d}} \in \Gamma\left(\operatorname{Hom}\left(\wedge^{2} \mathcal{G}_{0}, \mathcal{G}_{-1}\right)\right)$, such that we have

$$
\begin{aligned}
\dot{\mathfrak{j}}_{0}\left(\mathfrak{d}_{0}\right) & =\dot{\mathfrak{j}}_{1}\left(\mathfrak{d}_{1}\right), \\
\mathfrak{d}_{0} \circ \mathfrak{l}_{1} & =\mathfrak{l}_{1} \circ \mathfrak{d}_{1}, \\
\mathfrak{d}_{0} \mathfrak{l}_{2}(u, v) & =\mathfrak{l}_{2}\left(\mathfrak{d}_{0}(u), v\right)+\mathfrak{l}_{2}\left(u, \mathfrak{d}_{0}(v)\right)+\mathfrak{l}_{1} l_{\mathfrak{d}}(u, v), \\
\mathfrak{d}_{1} \mathfrak{l}_{2}(u, m) & =\mathfrak{l}_{2}\left(\mathfrak{d}_{0}(u), m\right)+\mathfrak{l}_{2}\left(u, \mathfrak{d}_{1}(m)\right)+l_{\mathfrak{d}}\left(u, \mathfrak{l}_{1}(m)\right),
\end{aligned}
$$

and

$$
\begin{aligned}
& l_{\mathfrak{d}}\left(u, \mathfrak{l}_{2}(v, w)\right)+\mathfrak{l}_{2}\left(u, l_{\mathfrak{d}}(v, w)\right)+\mathfrak{l}_{3}\left(\mathfrak{d}_{0}(u), v, w\right)+\mathfrak{l}_{3}\left(u, \mathfrak{d}_{0}(v), w\right)+\mathfrak{l}_{3}\left(u, v, \mathfrak{d}_{0}(w)\right) \\
= & \mathfrak{d}_{1} \mathfrak{l}_{3}(u, v, w)+l_{\mathfrak{d}}\left(\mathfrak{l}_{2}(u, v), w\right)+l_{\mathfrak{d}}\left(v, \mathfrak{l}_{2}(u, w)\right)+\mathfrak{l}_{2}\left(l_{\mathfrak{d}}(u, v), w\right)+\mathfrak{l}_{2}\left(v, l_{\mathfrak{d}}(u, w)\right),
\end{aligned}
$$

for all $u, v, w \in \Gamma\left(\mathcal{G}_{0}\right)$ and $m \in \Gamma\left(\mathcal{G}_{-1}\right)$.

It is obvious that the set of degree 0 derivations is the section space of a vector bundle, which we denote by $\operatorname{Der}^{0}(\mathfrak{G})$. Furthermore, we define $\operatorname{Der}^{-1}(\mathfrak{G})=\operatorname{Hom}\left(\mathcal{G}_{0}, \mathcal{G}_{-1}\right)$.

Example 3.6. For any $u \in \Gamma\left(\mathcal{G}_{0}\right)$, define $\operatorname{ad}_{u}^{0}: \Gamma\left(\mathcal{G}_{0}\right) \longrightarrow \Gamma\left(\mathcal{G}_{0}\right)$ and $\operatorname{ad}_{u}^{1}: \Gamma\left(\mathcal{G}_{-1}\right) \longrightarrow \Gamma\left(\mathcal{G}_{-1}\right)$ by

$$
\operatorname{ad}_{u}^{0} v=\mathfrak{l}_{2}(u, v), \quad \operatorname{ad}_{u}^{1} m=\mathfrak{l}_{2}(u, m), \quad \forall v \in \Gamma\left(\mathcal{G}_{0}\right), m \in \Gamma\left(\mathcal{G}_{-1}\right) .
$$

Define $l_{\mathrm{ad}_{u}}: \wedge^{2} \Gamma\left(\mathcal{G}_{0}\right) \longrightarrow \Gamma\left(\mathcal{G}_{-1}\right)$ by

$$
l_{\mathrm{ad}_{u}}(v, w)=\mathfrak{l}_{3}(u, v, w), \quad \forall v, w \in \Gamma\left(\mathcal{G}_{0}\right) .
$$

Then it is straightforward to see that $\left(\operatorname{ad}_{u}^{0}, \operatorname{ad}_{u}^{1}, l_{\mathrm{ad}_{u}}\right) \in \Gamma\left(\operatorname{Der}^{0}(\mathfrak{G})\right)$. 
Define $\mathfrak{j}: \operatorname{Der}^{0}(\mathfrak{G}) \longrightarrow T M$ by

$$
\dot{\mathfrak{j}}\left(\mathfrak{d}_{0}, \mathfrak{d}_{1}, l_{\mathfrak{d}}\right):=\dot{\mathfrak{j}}_{0}\left(\mathfrak{d}_{0}\right) .
$$

On the graded bundle $\operatorname{Der}(\mathfrak{G})=\operatorname{Der}^{0}(\mathfrak{G}) \oplus \operatorname{Der}^{-1}(\mathfrak{G})$, we define $\delta: \operatorname{Der}^{-1}(\mathfrak{G}) \longrightarrow \operatorname{Der}^{0}(\mathfrak{G})$ and skew-symmetric bracket operation $[\cdot, \cdot]$ Der $: \Gamma\left(\operatorname{Der}^{-i}(\mathfrak{G})\right) \times \Gamma\left(\operatorname{Der}^{-j}(\mathfrak{G})\right) \longrightarrow \Gamma\left(\operatorname{Der}^{-(i+j)}(\mathfrak{G})\right)$, $0 \leq i+j \leq 1$, by

$$
\begin{aligned}
\delta(\phi) & =\left(\mathfrak{l}_{1} \circ \phi, \phi \circ \mathfrak{l}_{1}, l_{\delta(\phi)}\right), \\
{\left[\left(\mathfrak{d}_{0}, \mathfrak{d}_{1}, l_{\mathfrak{d}}\right),\left(\mathfrak{t}_{0}, \mathfrak{t}_{1}, l_{\mathfrak{t}}\right)\right]_{\text {Der }} } & =\left(\left[\mathfrak{d}_{0}, \mathfrak{t}_{0}\right]_{C},\left[\mathfrak{d}_{1}, \mathfrak{t}_{1}\right]_{C}, l_{[\mathfrak{d}, \mathfrak{t}]_{C}}\right), \\
{\left[\left(\mathfrak{d}_{0}, \mathfrak{d}_{1}, l_{\mathfrak{o}}\right), \phi\right]_{\text {Der }} } & =\mathfrak{d}_{1} \circ \phi-\phi \circ \mathfrak{d}_{0},
\end{aligned}
$$

where $l_{\delta(\phi)}, l_{[\mathfrak{d}, \mathfrak{t}]_{C}} \in \Gamma\left(\operatorname{Hom}\left(\wedge^{2} \mathcal{G}_{0}, \mathcal{G}_{-1}\right)\right)$ are defined by

$$
\begin{aligned}
l_{\delta(\phi)}(u, v) & =\phi\left(\mathfrak{l}_{2}(u, v)\right)-\mathfrak{l}_{2}(\phi(u), v)-\mathfrak{l}_{2}(u, \phi(v)), \\
l_{\left[\mathfrak{d}, \mathfrak{t}_{C}\right.}(u, v) & =\mathfrak{d}_{1} l_{\mathfrak{t}}(u, v)-l_{\mathfrak{t}}\left(\mathfrak{d}_{0}(u), v\right)-l_{\mathfrak{t}}\left(u, \mathfrak{d}_{0}(v)\right)+l_{\mathfrak{d}}\left(\mathfrak{t}_{0}(u), v\right)+l_{\mathfrak{d}}\left(u, \mathfrak{t}_{0}(v)\right)-\mathfrak{t}_{1} l_{\mathfrak{d}}(u, v) .
\end{aligned}
$$

It is straightforward to deduce that

Proposition 3.7. Let $\mathfrak{G}=\left(\mathcal{G}_{-1} \oplus \mathcal{G}_{0} ; \mathfrak{l}_{1}, \mathfrak{l}_{2}, \mathfrak{l}_{3}\right)$ be a graded bundle of Lie 2-algebras. Then $\left(\operatorname{Der}^{-1}(\mathfrak{G}) \oplus \operatorname{Der}^{0}(\mathfrak{G}) ; \mathfrak{j}, \delta,[\cdot, \cdot]\right.$ Der $)$ is a strict Lie 2-algebroid.

We go on constructing a strict Lie 3-algebroid. On the 3-term graded vector bundles

$$
\operatorname{DER}(\mathfrak{G})=\mathcal{G}_{-1} \oplus\left(\operatorname{Der}^{-1}(\mathfrak{G}) \oplus \mathcal{G}_{0}\right) \oplus \operatorname{Der}^{0}(\mathfrak{G}),
$$

where the degree 0 part $\operatorname{DER}^{0}(\mathfrak{G})$ is $\operatorname{Der}^{0}(\mathfrak{G})$, the degree -1 part $\operatorname{DER}^{-1}(\mathfrak{G})$ is $\operatorname{Der}^{-1}(\mathfrak{G}) \oplus \mathcal{G}_{0}$ and the degree -2 part $\operatorname{DER}^{-2}(\mathfrak{G})$ is $\mathcal{G}_{-1}$, we define $\mathrm{d}: \operatorname{DER}^{-i}(\mathfrak{G}) \longrightarrow \operatorname{DER}^{-i+1}(\mathfrak{G}), i=1,2$ and $[\cdot, \cdot]_{\operatorname{DER}}: \Gamma\left(\operatorname{DER}^{-i}(\mathfrak{G})\right) \times \Gamma\left(\operatorname{DER}^{-j}(\mathfrak{G})\right) \longrightarrow \Gamma\left(\operatorname{DER}^{-(i+j)}(\mathfrak{G})\right), 0 \leq i+j \leq 2$, by

$$
\begin{aligned}
\mathrm{d}(m) & =-\mathfrak{l}_{2}(m, \cdot)+\mathfrak{l}_{1}(m), \\
\mathrm{d}(\phi+u) & =\delta(\phi)+\left(\operatorname{ad}_{u}^{0}, \operatorname{ad}_{u}^{1}, l_{\mathrm{ad}_{u}}\right), \\
{\left[\left(\mathfrak{d}_{0}, \mathfrak{d}_{1}, l_{\mathfrak{d}}\right),\left(\mathfrak{t}_{0}, \mathfrak{t}_{1}, l_{\mathfrak{t}}\right)\right]_{\text {DER }} } & =\left[\left(\mathfrak{d}_{0}, \mathfrak{d}_{1}, l_{\mathfrak{d}}\right),\left(\mathfrak{t}_{0}, \mathfrak{t}_{1}, l_{\mathfrak{t}}\right)\right]_{\text {Der }}, \\
{\left[\left(\mathfrak{d}_{0}, \mathfrak{d}_{1}, l_{\mathfrak{d}}\right), \phi+u\right]_{\text {DER }} } & =\left[\left(\mathfrak{d}_{0}, \mathfrak{d}_{1}, l_{\mathfrak{d}}\right), \phi\right]_{\text {Der }}+l_{\mathfrak{d}}(u, \cdot)+\mathfrak{d}_{0}(u), \\
{\left[\left(\mathfrak{d}_{0}, \mathfrak{d}_{1}, l_{\mathfrak{d}}\right), m\right]_{\text {DER }} } & =\mathfrak{d}_{1}(m), \\
{[\phi+u, \psi+v]_{\text {DER }} } & =\phi(v)+\psi(u),
\end{aligned}
$$

for all $\left(\mathfrak{d}_{0}, \mathfrak{d}_{1}, l_{\mathfrak{d}}\right),\left(\mathfrak{t}_{0}, \mathfrak{t}_{1}, l_{\mathfrak{t}}\right) \in \Gamma\left(\operatorname{Der}^{0}(\mathfrak{G})\right), \phi, \psi \in \Gamma\left(\operatorname{Der}^{-1}(\mathfrak{G})\right), u, v \in \Gamma\left(\mathcal{G}_{0}\right)$ and $m \in \Gamma\left(\mathcal{G}_{-1}\right)$.

Theorem 3.8. Let $\mathfrak{G}=\left(\mathcal{G}_{-1} \oplus \mathcal{G}_{0} ; \mathfrak{l}_{1}, \mathfrak{l}_{2}, \mathfrak{l}_{3}\right)$ be a graded bundle of Lie 2-algebras. Then

$$
\left(\operatorname{DER}^{-2}(\mathfrak{G}) \oplus \operatorname{DER}^{-1}(\mathfrak{G}) \oplus \operatorname{DER}^{0}(\mathfrak{G}) ; \mathfrak{j}, \mathrm{d},[\cdot, \cdot] \text { DER }\right)
$$

is a strict Lie 3-algebroid.

Proof. The proof is a straightforward verification. We leave it to readers.

With above preparations, we go back to Subsection 3.1. For a transitive Lie 2-algebroid $\left(A_{-1} \oplus\right.$ $\left.A_{0} ; \rho, l_{1}, l_{2}, l_{3}\right)$, let $\mathfrak{G}=\left(A_{-1} \oplus \mathcal{G} ; \mathfrak{l}_{1}, \mathfrak{l}_{2}, \mathfrak{l}_{3}\right)$ be the corresponding graded bundle of Lie 2-algebras, where $\mathcal{G}=\operatorname{ker}(\rho)$, and $\operatorname{DER}(\mathfrak{G})$ the strict Lie 3 -algebroid given above. 
Define $F^{1}: T M \longrightarrow \operatorname{DER}^{0}(\mathfrak{G})$ by

$$
F^{1}(X)=\left(\nabla_{X}^{0}, \nabla_{X}^{1}, K(X, \cdot, \cdot)\right), \quad \forall X \in \mathfrak{X}(M) .
$$

By (13), (14), (15), (16), $F^{1}$ is well-defined.

Define $F^{2}: \wedge^{2} T M \longrightarrow \mathrm{DER}^{-1}(\mathfrak{G})$ by

$$
F^{2}(X, Y)=J(X, Y, \cdot)+R_{\gamma}(X, Y), \quad \forall X, Y \in \mathfrak{X}(M) .
$$

Define $F^{3}: \wedge^{3} T M \longrightarrow \mathrm{DER}^{-2}(\mathfrak{G})$ by

$$
F^{3}(X, Y, Z)=I_{\gamma}(X, Y, Z), \quad \forall X, Y, Z \in \mathfrak{X}(M) .
$$

Theorem 3.9. Let $\left(A_{-1} \oplus A_{0} ; \rho, l_{1}, l_{2}, l_{3}\right)$ be a transitive Lie 2-algebroid. Then $\left(F^{1}, F^{2}, F^{3}\right)$ is a morphism from the Lie algebroid TM to the strict Lie 3-algebroid DER(G).

Proof. We only give a sketch of the proof and leave details to readers. By (17), (18) and (23), we deduce that

$$
F^{1}([X, Y])-\left[F^{1}(X), F^{1}(Y)\right]_{\mathrm{DER}}=\mathrm{d} F^{2}(X, Y), \quad \forall X, Y \in \mathfrak{X}(M) .
$$

By (19) and (20), we deduce that

$$
\left[F^{1}(X), F^{2}(Y, Z)\right]_{\mathrm{DER}}-F^{2}([X, Y], Z)+c . p .=\mathrm{d} F^{3}(X, Y, Z) .
$$

By (21), we deduce that

$$
\begin{aligned}
& \sum_{i=1}^{4}(-1)^{i+1}\left[F^{1}\left(X_{i}\right), F^{3}\left(X_{1}, \cdots, \widehat{X}_{i}, \cdots X_{4}\right)\right]_{\mathrm{DER}} \\
& +\sum_{i<j}(-1)^{i+j}\left(F^{3}\left(\left[X_{i}, X_{j}\right], X_{k}, X_{l}\right)-\frac{1}{2}\left[F^{2}\left(X_{i}, X_{j}\right), F^{2}\left(X_{k}, X_{l}\right)\right]_{\mathrm{DER}}\right) \\
= & \left(d_{\nabla^{1}} I_{\gamma}+J \circ R_{\gamma}\right)\left(X_{1}, \cdots, X_{4}\right) \\
= & 0 .
\end{aligned}
$$

Thus, $\left(F^{1}, F^{2}, F^{3}\right)$ is a morphism form $T M$ to $\operatorname{DER}(\mathfrak{G})$.

\section{The first Pontryagin class of a quadratic Lie 2-algebroid}

In this section, we give the notion of a quadratic Lie 2-algebroid and define its first Pontryagin class, which is a cohomology class in $H^{5}(M)$.

Definition 4.1. A transitive Lie 2-algebroid $\left(A_{-1} \oplus A_{0} ; \rho, l_{1}, l_{2}, l_{3}\right)$ is said to be a quadratic Lie 2 -algebroid if there is a degree 1 nondegenerate graded symmetric bilinear form $\mathcal{S}$ on the graded vector bundle $A_{-1} \oplus \operatorname{ker}(\rho)$ satisfying

$$
\begin{aligned}
\mathcal{S}\left(\mathfrak{l}_{1}(m), p\right) & =\mathcal{S}\left(m, \mathfrak{l}_{1}(p)\right), \\
\rho\left(e^{0}\right) \mathcal{S}(u, m) & =\mathcal{S}\left(l_{2}\left(e^{0}, u\right), m\right)+\mathcal{S}\left(u, l_{2}\left(e^{0}, m\right)\right), \\
\mathcal{S}\left(l_{3}\left(e_{1}^{0}, e_{2}^{0}, u\right), v\right) & =-\mathcal{S}\left(l_{3}\left(e_{1}^{0}, e_{2}^{0}, v\right), u\right),
\end{aligned}
$$

for all $u, v \in \Gamma(\operatorname{ker}(\rho)), e^{0}, e_{1}^{0}, e_{2}^{0} \in \Gamma\left(A_{0}\right)$ and $m, p \in \Gamma\left(A_{-1}\right)$. 
Let $\left(A_{-1} \oplus A_{0} ; \rho, l_{1}, l_{2}, l_{3}\right)$ be a quadratic Lie 2 -algebroid. As before, denote by $\mathcal{G}=\operatorname{ker}(\rho)$. By the nondegeneracy of $\mathcal{S}$, we deduce that $A_{-1} \cong \mathcal{G}^{*}$ and we will write $A_{-1}=\mathcal{G}^{*}$ directly. Then the pairing $\mathcal{S}$ is simply given by

$$
\mathcal{S}(u+m, v+p)=\langle u, p\rangle+\langle v, m\rangle, \quad \forall u, v \in \Gamma(\mathcal{G}), m, p \in \Gamma\left(\mathcal{G}^{*}\right) .
$$

By (30)-(32), we deduce that for the graded bundle of Lie 2-algebras $\left(\mathcal{G}^{*} \oplus \mathcal{G} ; \mathfrak{l}_{1}, \mathfrak{l}_{2}, \mathfrak{l}_{3}\right)$, there holds:

$$
\begin{aligned}
\left\langle\mathfrak{l}_{1}(m), p\right\rangle & =\left\langle m, \mathfrak{l}_{1}(p)\right\rangle, \quad \forall m, p \in \Gamma\left(\mathcal{G}^{*}\right), \\
\left\langle\mathfrak{l}_{2}(u, v), m\right\rangle & =-\left\langle v, \mathfrak{l}_{2}(u, m)\right\rangle, \quad \forall u, v \in \Gamma(\mathcal{G}), m \in \Gamma\left(\mathcal{G}^{*}\right), \\
\left\langle\mathfrak{l}_{3}(u, v, w), x\right\rangle & =-\left\langle w, \mathfrak{l}_{3}(u, v, x)\right\rangle, \quad \forall u, v, w, x \in \Gamma(\mathcal{G}) .
\end{aligned}
$$

Proposition 4.2. With the same assumption and conditions in Theorem 3.3, the transitive Lie 2-algebroid $\left(\mathcal{G}^{*} \oplus(\mathcal{G} \oplus T M) ; \rho, l_{1}, l_{2}, l_{3}\right)$ is a quadratic Lie 2 -algebroid, where $\rho, l_{1}, l_{2}, l_{3}$ are given by (12) for totally skew-symmetric I, $J, K$, if and only if (34)-(36) and the following equalities hold:

$$
\begin{aligned}
\left\langle\nabla_{X}^{0} u, m\right\rangle+\left\langle u, \nabla_{X}^{1} m\right\rangle & =X\langle u, m\rangle, \\
\langle J(X, Y, u), v\rangle+\langle J(X, Y, v), u\rangle & =0 \\
\langle K(X, u, v), w\rangle+\langle K(X, u, w), v\rangle & =0 .
\end{aligned}
$$

Proof. It follows from that the invariant conditions in the definition of a quadratic Lie 2-algebroid is equivalent to (34)-(39).

Let $\left(\mathcal{G}^{*} \oplus(\mathcal{G} \oplus T M) ; \rho, l_{1}, l_{2}, l_{3}\right)$ be a quadratic Lie 2-algebroid given by (12) with $\mathcal{S}$ the graded symmetric bilinear form given by (33). Define $\mathcal{S}\left(R_{\gamma}, I_{\gamma}\right) \in \Omega^{5}(M)$ by

$$
\mathcal{S}\left(R_{\gamma}, I_{\gamma}\right)\left(X_{1}, \cdots, X_{5}\right)=\frac{1}{12} \sum_{\tau \in S_{5}} \operatorname{sgn}(\tau) \mathcal{S}\left(R_{\gamma}\left(X_{\tau(1)}, X_{\tau(2)}\right), I_{\gamma}\left(X_{\tau(3)}, X_{\tau(4)}, X_{\tau(5)}\right)\right) .
$$

Theorem 4.3. The 5 -form $\mathcal{S}\left(R_{\gamma}, I_{\gamma}\right)$ is closed, i.e. $d \mathcal{S}\left(R_{\gamma}, I_{\gamma}\right)=0$, and its cohomology class $\left[\mathcal{S}\left(R_{\gamma}, I_{\gamma}\right)\right]$ in $H^{5}(M)$ does not depend on the choices of $\sigma$ and $\gamma$.

The cohomology class $\left[\mathcal{S}\left(R_{\gamma}, I_{\gamma}\right)\right] \in H^{5}(M)$ is called the first Pontryagin class of the quadratic Lie 2-algebroid $\left(A_{-1} \oplus A_{0} ; \rho, l_{1}, l_{2}, l_{3}\right)$. To prove the above theorem, we need the following technical lemma.

Lemma 4.4. For all $\Xi \in \Gamma\left(\operatorname{Hom}\left(\wedge^{k} T M, \mathcal{G}\right)\right)$ and $\Pi \in \Gamma\left(\operatorname{Hom}\left(\wedge^{l} T M, \mathcal{G}^{*}\right)\right)$, we have

$$
d \mathcal{S}(\Xi, \Pi)=\mathcal{S}\left(d_{\nabla^{0}} \Xi, \Pi\right)+(-1)^{k} \mathcal{S}\left(\Xi, d_{\nabla^{1}} \Pi\right),
$$

where $\mathcal{S}(\Xi, \Pi) \in \Omega^{k+l}(M)$ is defined by

$$
\mathcal{S}(\Xi, \Pi)\left(X_{1}, \cdots, X_{k+l}\right)=\frac{1}{k ! l !} \sum_{\tau \in S_{k+l}} \operatorname{sgn}(\tau) \mathcal{S}\left(\Xi\left(X_{\tau(1)}, \cdots, X_{\tau(k)}\right), \Pi\left(X_{\tau(k+1)}, \cdots, X_{\tau(k+l)}\right)\right),
$$

for all $X_{1}, \cdots, X_{k+l} \in \mathfrak{X}(M)$. 
Proof. First we have

$$
\begin{aligned}
& d \mathcal{S}(\Xi, \Pi)\left(X_{1}, \cdots, X_{k+l+1}\right) \\
= & \sum_{i=1}^{k+l+1}(-1)^{i+1} X_{i} \mathcal{S}(\Xi, \Pi)\left(X_{1}, \cdots, \hat{X}_{i}, \cdots, X_{k+l+1}\right) \\
& +\sum_{i<j}(-1)^{i+j} \mathcal{S}(\Xi, \Pi)\left(\left[X_{i}, X_{j}\right], X_{1}, \cdots, \hat{X}_{i}, \cdots, \hat{X}_{j}, \cdots, X_{k+l+1}\right) .
\end{aligned}
$$

Then by (37), we can deduce that (41) holds after a careful computation. We omit details.

The proof of Theorem 4.3; By Lemma 4.4, (19), (21), (34) and (38), we have

$$
\begin{aligned}
d \mathcal{S}\left(R_{\gamma}, I_{\gamma}\right) & =\mathcal{S}\left(d_{\nabla^{0}} R_{\gamma}, I_{\gamma}\right)+\mathcal{S}\left(R_{\gamma}, d_{\nabla^{1}} I_{\gamma}\right) \\
& =\mathcal{S}\left(\mathfrak{l}_{1} I_{\gamma}, I_{\gamma}\right)-\mathcal{S}\left(R_{\gamma}, J \circ R_{\gamma}\right) \\
& =0 .
\end{aligned}
$$

Thus, $\mathcal{S}\left(R_{\gamma}, I_{\gamma}\right)$ is a closed 5 -form.

Furthermore, by Lemma 4.4 and (19), we have

$$
\begin{aligned}
\mathcal{S}\left(R_{\gamma}, I_{\gamma}\right) & =\mathcal{S}(R, I)+\mathcal{S}\left(R, d_{\nabla^{1}} \gamma\right)+\mathcal{S}\left(\mathfrak{l}_{1} \circ \gamma, I\right)+\mathcal{S}\left(\mathfrak{l}_{1} \circ \gamma, d_{\nabla^{1}} \gamma\right) \\
& =\mathcal{S}(R, I)+d \mathcal{S}(R, \gamma)+\frac{1}{2} d \mathcal{S}\left(\mathfrak{l}_{1} \circ \gamma, \gamma\right)
\end{aligned}
$$

which implies that the cohomology class does not depend on the choices of $\gamma$.

If we choose another section $\sigma^{\prime}: T M \longrightarrow A_{0}$, then we define $\theta: T M \longrightarrow \mathcal{G}$ by

$$
\theta(X)=\sigma(X)-\sigma^{\prime}(X), \quad \forall X \in \mathfrak{X}(M) .
$$

We have

$$
\begin{aligned}
R^{\prime}(X, Y) & =\sigma^{\prime}[X, Y]-l_{2}\left(\sigma^{\prime}(X), \sigma^{\prime}(Y)\right)-\mathfrak{l}_{1} \circ \gamma(X, Y) \\
& =R(X, Y)+d_{\nabla^{0}} \theta(X, Y)-\mathfrak{l}_{2}(\theta(X), \theta(Y))
\end{aligned}
$$

and

$$
\begin{aligned}
I^{\prime}(X, Y, Z) & =-l_{3}\left(\sigma^{\prime}(X), \sigma^{\prime}(Y), \sigma^{\prime}(Z)\right)-d_{\nabla^{1}} \gamma(X, Y, Z) \\
& =I(X, Y, Z)+\mathfrak{l}_{3}(\theta(X), \theta(Y), \theta(Z))-(J(X, Y, \theta(Z))+K(X, \theta(Y), \theta(Z))+\text { c.p. })
\end{aligned}
$$

We define $\mathfrak{l}_{2} \circ \theta: \wedge^{2} T M \longrightarrow \mathcal{G}, \mathfrak{l}_{3} \circ \theta: \wedge^{3} T M \longrightarrow \mathcal{G}^{*}, J \circ \theta: \wedge^{3} T M \longrightarrow \mathcal{G}^{*}$ and $K \circ \theta: \wedge^{3} T M \longrightarrow \mathcal{G}^{*}$ respectively by

$$
\begin{aligned}
\mathfrak{l}_{2} \circ \theta(X, Y) & =\mathfrak{l}_{2}(\theta(X), \theta(Y)), \\
\mathfrak{l}_{3} \circ \theta(X, Y, Z) & =\mathfrak{l}_{3}(\theta(X), \theta(Y), \theta(Z)), \\
J \circ \theta(X, Y, Z) & =J(\theta(X), Y, Z)+J(X, \theta(Y), Z)+J(X, Y, \theta(Z)), \\
K \circ \theta(X, Y, Z) & =K(X, \theta(Y), \theta(Z))+K(\theta(X), Y, \theta(Z))+K(\theta(X), \theta(Y), Z) .
\end{aligned}
$$


Therefore, by the following Lemma 4.5.Lemma 4.9, we have

$$
\begin{aligned}
\mathcal{S}\left(R_{\gamma}^{\prime}, I_{\gamma}^{\prime}\right)= & \mathcal{S}\left(R_{\gamma}+d_{\nabla^{0}} \theta-\mathfrak{l}_{2} \circ \theta, I_{\gamma}-J \circ \theta-K \circ \theta+\mathfrak{l}_{3} \circ \theta\right) \\
= & \mathcal{S}\left(R_{\gamma}, I_{\gamma}\right)+\mathcal{S}\left(d_{\nabla^{0}} \theta, I_{\gamma}\right)-\mathcal{S}\left(R_{\gamma}, J \circ \theta\right)-\mathcal{S}\left(R_{\gamma}, K \circ \theta\right)+\mathcal{S}\left(R_{\gamma}, \mathfrak{l}_{3} \circ \theta\right) \\
& -\mathcal{S}\left(d_{\nabla^{0}} \theta, J \circ \theta\right)-\mathcal{S}\left(d_{\nabla^{0}} \theta, K \circ \theta\right)+\mathcal{S}\left(d_{\nabla^{0}} \theta, \mathfrak{l}_{3} \circ \theta\right) \\
& -\mathcal{S}\left(\mathfrak{l}_{2} \circ \theta, I_{\gamma}\right)+\mathcal{S}\left(\mathfrak{l}_{2} \circ \theta, J \circ \theta\right)+\mathcal{S}\left(\mathfrak{l}_{2} \circ \theta, K \circ \theta\right)-\mathcal{S}\left(\mathfrak{l}_{2} \circ \theta, \mathfrak{l}_{3} \circ \theta\right) \\
= & \mathcal{S}\left(R_{\gamma}, I_{\gamma}\right)+d\left(C_{4}^{I}-C_{4}^{J}-C_{4}^{K}+C_{4}^{\mathfrak{l}_{3}}\right),
\end{aligned}
$$

which implies that $\mathcal{S}\left(R_{\gamma}^{\prime}, I_{\gamma}^{\prime}\right)$ and $\mathcal{S}\left(R_{\gamma}, I_{\gamma}\right)$ are in the same cohomology class.

For any $\mathcal{G}$-valued 1-form $\theta \in \Gamma(\operatorname{Hom}(T M, \mathcal{G}))$, we define a 4 -form $C_{4}^{I} \in \Omega^{4}(M)$ by

$$
C_{4}^{I}=\mathcal{S}\left(\theta, I_{\gamma}\right)
$$

Lemma 4.5. With the above notations, we have

$$
d C_{4}^{I}=\mathcal{S}\left(d_{\nabla^{0}} \theta, I_{\gamma}\right)-\mathcal{S}\left(R_{\gamma}, J \circ \theta\right) .
$$

Proof. By Lemma 4.4, (21) and (38), we have

$$
\begin{aligned}
d \mathcal{S}\left(\theta, I_{\gamma}\right) & =\mathcal{S}\left(d_{\nabla^{0}} \theta, I_{\gamma}\right)-\mathcal{S}\left(\theta, d_{\nabla^{1}} I_{\gamma}\right) \\
& =\mathcal{S}\left(d_{\nabla^{0}} \theta, I_{\gamma}\right)+\mathcal{S}\left(\theta, J \circ R_{\gamma}\right) \\
& =\mathcal{S}\left(d_{\nabla^{0}} \theta, I_{\gamma}\right)-\mathcal{S}\left(R_{\gamma}, J \circ \theta\right) .
\end{aligned}
$$

The proof is finished.

For any $\mathcal{G}$-valued 1-form $\theta \in \Gamma(\operatorname{Hom}(T M, \mathcal{G}))$, we define a 4 -form $C_{4}^{J} \in \Omega^{4}(M)$ by

$$
C_{4}^{J}=\frac{1}{2} \mathcal{S}(\theta, J \circ \theta)
$$

Lemma 4.6. With the above notations, we have

$$
d C_{4}^{J}=\mathcal{S}\left(d_{\nabla^{\circ}} \theta, J \circ \theta\right)+\mathcal{S}\left(R_{\gamma}, K \circ \theta\right)+\mathcal{S}\left(\mathfrak{l}_{2} \circ \theta, I_{\gamma}\right) .
$$

Proof. By Lemma 4.4, (20), (38), (39) and (35), we obtain

$$
\begin{aligned}
d C_{4}^{J} & =\frac{1}{2} d \mathcal{S}(\theta, J \circ \theta) \\
& =\frac{1}{2}\left(\mathcal{S}\left(d_{\nabla^{0}} \theta, J \circ \theta\right)-\mathcal{S}\left(\theta, d_{\nabla^{1}}(J \circ \theta)\right)\right) \\
& =\frac{1}{2}\left(\mathcal{S}\left(d_{\nabla^{0}} \theta, J \circ \theta\right)+\mathcal{S}\left(d_{\nabla^{0}} \theta, J \circ \theta\right)+2 \mathcal{S}\left(R_{\gamma}, K \circ \theta\right)+2 \mathcal{S}\left(\mathfrak{l}_{2} \circ \theta, I_{\gamma}\right)\right) \\
& =\mathcal{S}\left(d_{\nabla^{0}} \theta, J \circ \theta\right)+\mathcal{S}\left(R_{\gamma}, K \circ \theta\right)+\mathcal{S}\left(\mathfrak{l}_{2} \circ \theta, I_{\gamma}\right) .
\end{aligned}
$$

The proof is finished.

For any $\mathcal{G}$-valued 1 -form $\theta \in \Gamma(\operatorname{Hom}(T M, \mathcal{G}))$, we define a 4 -form $C_{4}^{K} \in \Omega^{4}(M)$ by

$$
C_{4}^{K}=\frac{1}{3} \mathcal{S}(\theta, K \circ \theta)
$$


Lemma 4.7. With the above notations, we have

$$
d C_{4}^{K}=\mathcal{S}\left(d_{\nabla^{0}} \theta, K \circ \theta\right)-\mathcal{S}\left(R_{\gamma}, \mathfrak{l}_{3} \circ \theta\right)-\mathcal{S}\left(\mathfrak{l}_{2} \circ \theta, J \circ \theta\right) .
$$

Proof. By Lemma 4.4, (23), (38), (39), (35) and (36), we have

$$
\begin{aligned}
d C_{4}^{K} & =\frac{1}{3} d \mathcal{S}(\theta, K \circ \theta) \\
& =\frac{1}{3}\left(\mathcal{S}\left(d_{\nabla^{0}} \theta, K \circ \theta\right)-\mathcal{S}\left(\theta, d_{\nabla^{1}}(K \circ \theta)\right)\right) \\
& =\frac{1}{3}\left(\mathcal{S}\left(d_{\nabla^{0}} \theta, K \circ \theta\right)+2 \mathcal{S}\left(d_{\nabla^{0}} \theta, K \circ \theta\right)-3 \mathcal{S}\left(R_{\gamma}, \mathfrak{l}_{3} \circ \theta\right)-3 \mathcal{S}\left(\mathfrak{l}_{2} \circ \theta, J \circ \theta\right)\right) \\
& =\mathcal{S}\left(d_{\nabla^{0}} \theta, K \circ \theta\right)-\mathcal{S}\left(R_{\gamma}, \mathfrak{l}_{3} \circ \theta\right)-\mathcal{S}\left(\mathfrak{l}_{2} \circ \theta, J \circ \theta\right) .
\end{aligned}
$$

The proof is finished.

For any $\mathcal{G}$-valued 1 -form $\theta \in \Gamma(\operatorname{Hom}(T M, \mathcal{G}))$, we define a 4 -form $C_{4}^{\mathfrak{l}_{3}} \in \Omega^{4}(M)$ by

$$
C_{4}^{\mathfrak{l}_{3}}=\frac{1}{4} \mathcal{S}\left(\theta, \mathfrak{l}_{3} \circ \theta\right)
$$

Equivalently,

$$
C_{4}^{\mathfrak{l}_{3}}\left(X_{1}, X_{2}, X_{3}, X_{4}\right)=\mathcal{S}\left(\theta\left(X_{1}\right), \mathfrak{l}_{3}\left(\theta\left(X_{2}\right), \theta\left(X_{3}\right), \theta\left(X_{4}\right)\right)\right) .
$$

Lemma 4.8. With the above notations, we have

$$
d C_{4}^{\mathfrak{l}_{3}}=\mathcal{S}\left(d_{\nabla^{0}} \theta, \mathfrak{l}_{3} \circ \theta\right)+\mathcal{S}\left(\mathfrak{l}_{2} \circ \theta, K \circ \theta\right) .
$$

Proof. By Lemma 4.4, (16), (36) and (39), we have

$$
\begin{aligned}
d C_{4}^{\mathfrak{l}_{3}} & =\frac{1}{4} d \mathcal{S}\left(\theta, \mathfrak{l}_{3} \circ \theta\right) \\
& =\frac{1}{4}\left(\mathcal{S}\left(d_{\nabla^{0}} \theta, \mathfrak{l}_{3} \circ \theta\right)-\mathcal{S}\left(\theta, d_{\nabla^{1}} \mathfrak{l}_{3} \circ \theta\right)\right) \\
& =\frac{1}{4}\left(\mathcal{S}\left(d_{\nabla^{0}} \theta, \mathfrak{l}_{3} \circ \theta\right)+3 \mathcal{S}\left(d_{\nabla^{0}} \theta, \mathfrak{l}_{3} \circ \theta\right)+4 \mathcal{S}\left(\mathfrak{l}_{2} \circ \theta, K \circ \theta\right)\right) \\
& =\mathcal{S}\left(d_{\nabla^{0}} \theta, \mathfrak{l}_{3} \circ \theta\right)+\mathcal{S}\left(\mathfrak{l}_{2} \circ \theta, K \circ \theta\right) .
\end{aligned}
$$

The proof is finished.

Lemma 4.9. With the above notations, we have

$$
\mathcal{S}\left(\mathfrak{l}_{2} \circ \theta, \mathfrak{l}_{3} \circ \theta\right)=0 .
$$

Proof. It follows from (35), (36) and the Jacobiator identity for $\mathfrak{l}_{3}$.

\section{Exact CLWX 2-algebroids}

A CLWX 2-algebroid $\left(E_{-1}, E_{0}, \partial, \rho, S, \diamond, \Omega\right)$ is called exact if we have the following exact sequence of vector bundles:

$$
0 \longrightarrow T^{*} M \stackrel{\rho^{*}}{\longrightarrow} E_{-1} \stackrel{\partial}{\longrightarrow} E_{0} \stackrel{\rho}{\longrightarrow} T M \longrightarrow 0,
$$

where $\rho^{*}: T^{*} M \longrightarrow E_{-1}$ is defined by

$$
S\left(\rho^{*}(\alpha), e^{0}\right)=\left\langle\alpha, \rho\left(e^{0}\right)\right\rangle, \quad \forall \alpha \in \Omega^{1}(M), e^{0} \in \Gamma\left(E_{0}\right) .
$$




\subsection{Skeletal exact CLWX 2-algebroids}

In this subsection, we associate a skeletal exact CLWX 2-algebroid a cohomology class in $H^{4}(M)$, can call it the higher analogue of the Ševera class.

Definition 5.1. An exact CLWX 2-algebroid $\left(E_{-1}, E_{0}, \partial, \rho, S, \diamond, \Omega\right)$ is called skeletal if $\partial=0$.

For a skeletal exact CLWX 2-algebroid $\left(E_{-1}, E_{0}, \partial, \rho, S, \diamond, \Omega\right)$, by the exactness, we have

$$
E_{-1} \cong T^{*} M, \quad E_{0} \cong T M .
$$

In the sequel, we will write $E_{-1}=T^{*} M, E_{0}=T M$ directly. By definition, $\partial=0 . S$ is given by

$$
S(X+\alpha, Y+\beta)=\langle\alpha, Y\rangle+\langle\beta, X\rangle, \quad \forall X, Y \in \mathfrak{X}(M), \alpha, \beta \in \Omega^{1}(M) .
$$

Obviously, we have

$$
X \diamond Y=[X, Y] .
$$

By Axiom (iv) in Definition 2.5 we have

$$
\begin{aligned}
X\langle\alpha, Y\rangle & =X S(\alpha, Y)=S(X \diamond \alpha, Y)+S(\alpha, X \diamond Y) \\
& =\langle X \diamond \alpha, Y\rangle+\langle\alpha,[X, Y]\rangle,
\end{aligned}
$$

which implies that

$$
X \diamond \alpha=L_{X} \alpha .
$$

Then by Axiom (ii), we deduce that

$$
\alpha \diamond X=-\iota_{X} d \alpha
$$

Finally, by Axiom (v), $\Omega: \wedge^{3} \mathfrak{X}(M) \longrightarrow \Omega^{1}(M)$ gives rise to a 4 -form $H \in \Omega^{4}(M)$ by

$$
H(X, Y, Z, W)=S(\Omega(X, Y, Z), W) .
$$

By Axiom (i), $H$ is closed. Summarize the above discussion, we have

Proposition 5.2. Any skeletal exact CLWX 2-algebroid must be of the form $\left(T^{*}[1] M, T M, \partial=\right.$ $0, \rho=\mathrm{id}, S, \diamond, \Omega)$, where $S, \diamond$ are given by (51), (52), (53), (54), and $\Omega$ is equivalent to a closed 4-form $H$.

We will denote a skeletal exact CLWX 2-algebroid simply by $\left(T^{*}[1] M, T M, S, \diamond, H\right)$.

Definition 5.3. An isomorphism from a CLWX 2-algebroid $\left(E_{-1}, E_{0}, \partial, \rho, S, \diamond, \Omega\right)$ to a CLWX 2-algebroid $\left(E_{-1}^{\prime}, E_{0}^{\prime}, \partial^{\prime}, \rho^{\prime}, S^{\prime}, \diamond^{\prime}, \Omega^{\prime}\right)$ consists of two bundle isomorphisms $\Phi_{0}: E_{0} \longrightarrow E_{0}^{\prime}, \Phi_{1}$ : $E_{-1} \longrightarrow E_{-1}^{\prime}$ and a bundle morphism $\Phi_{2}: \wedge^{2} E_{0} \longrightarrow E_{-1}^{\prime}$ such that

(i) $\rho^{\prime} \circ \Phi_{0}=\rho$,

(ii) $S\left(\Phi_{0}\left(e^{0}\right), \Phi_{1}\left(e^{1}\right)\right)=S\left(e^{0}, e^{1}\right)$, for all $e^{0} \in \Gamma\left(E_{0}\right)$ and $e^{1} \in \Gamma\left(E_{-1}\right)$,

(ii) $\left(\Phi_{0}, \Phi_{1}, \Phi_{2}\right)$ is an isomorphism from the Leibniz 2-algebra $\left(\Gamma\left(E_{-1}\right) \oplus \Gamma\left(E_{0}\right) ; \partial, \diamond, \Omega\right)$ to the Leibniz 2-algebra $\left(\Gamma\left(E_{-1}^{\prime}\right) \oplus \Gamma\left(E_{0}^{\prime}\right) ; \partial^{\prime}, \diamond^{\prime}, \Omega^{\prime}\right)$. 
We have seen that different skeletal exact CLWX 2-algebroids are differed by closed 4-forms. Now we show that if two closed 4-forms $H$ and $H^{\prime}$ are in the same cohomology class, the corresponding skeletal exact CLWX 2-algebroids are isomorphic. Thus, skeletal exact CLWX 2-algebroids are classified by $H^{4}(M)$. This is a higher analogous result of that exact Courant algebroids are classified by $H^{3}(M)$.

Theorem 5.4. Let $\left(T^{*}[1] M, T M, S, \diamond, H\right)$ and $\left(T^{*}[1] M, T M, S, \diamond, H^{\prime}\right)$ be two skeletal exact CLWX 2-algebroids. They are isomorphic if and only if $H$ and $H^{\prime}$ are in the same cohomology class.

Proof. If $H$ and $H^{\prime}$ are in the same cohomology class, we assume that $H=H^{\prime}+d h$. Then $\left(\Phi_{0}=\right.$ $\left.\mathrm{id}, \Phi_{1}=\mathrm{id}, \Phi_{2}=h\right)$ gives the required isomorphism from $\left(T^{*}[1] M, T M, S, \diamond, H\right)$ to $\left(T^{*}[1] M, T M, S, \diamond, H^{\prime}\right)$. In fact, what we need to show is that $\left(\Phi_{0}=\mathrm{id}, \Phi_{1}=\mathrm{id}, \Phi_{2}=h\right)$ is a Leibniz 2-algebra morphism from the Leibniz 2-algebra $\left(\Omega^{1}(M), \mathfrak{X}(M), \partial=0, \diamond, H\right)$ to $\left(\Omega^{1}(M), \mathfrak{X}(M), \partial=0, \diamond, H^{\prime}\right)$. The only nontrivial thing is to verify that

$$
X \diamond h(Y, Z)-Y \diamond h(X, Z)+h(X, Y) \diamond Z+H^{\prime}(X, Y, Z)=h([X, Y], Z)+c . p .+H(X, Y, Z),
$$

which is equivalent to $d h+H^{\prime}=H$.

It is known that exact Courant algebroids are classified by the Ševera class 24. Thus, for a skeletal exact CLWX 2-algebroid $\left(T^{*}[1] M, T M, S, \diamond, H\right)$, we call the cohomology class $[H] \in H^{4}(M)$ the higher analogue of the Ševera class.

\subsection{CLWX-extension of a quadratic Lie 2-algebroid}

In this subsection, we show that every exact CLWX 2-algebroid gives rise to a quadratic Lie 2-algebroid and a quadratic Lie 2-algebroid admits a CLWX-extension if and only if its first Pontryagin class vanishes.

Let $\left(E_{-1}, E_{0}, \partial, \rho, S, \diamond, \Omega\right)$ be an exact CLWX 2-algebroid. Denote by $F_{-1}=E_{-1} / \rho^{*}\left(T^{*}[1] M\right)$ and we have the following short exact sequence:

$$
0 \longrightarrow T^{*}[1] M \stackrel{\rho^{*}}{\longrightarrow} E_{-1} \stackrel{\mathrm{pr}}{\longrightarrow} F_{-1} \longrightarrow 0 .
$$

On the graded vector bundle $F_{-1} \oplus E_{0}$, define $l_{1}: F_{-1} \longrightarrow E_{0}$ by

$$
l_{1}(m)=\partial(\widetilde{m}), \quad \forall m \in \Gamma\left(F_{-1}\right), \tilde{m} \in \Gamma\left(E_{-1}\right) \text { such that } \operatorname{pr}(\widetilde{m})=m ;
$$

define $l_{2}$ by

$$
l_{2}\left(e_{1}^{0}, e_{2}^{0}\right)=e_{1}^{0} \diamond e_{2}^{0}, \quad l_{2}\left(e^{0}, m\right)=\operatorname{pr}\left(e^{0} \diamond \widetilde{m}\right), \quad l_{2}\left(m, e^{0}\right)=\operatorname{pr}\left(\widetilde{m} \diamond e^{0}\right) ;
$$

and define $l_{3}: \wedge^{3} \Gamma\left(E_{0}\right) \longrightarrow \Gamma\left(F_{-1}\right)$ by

$$
l_{3}\left(e_{1}^{0}, e_{2}^{0}, e_{3}^{0}\right)=\operatorname{pr} \Omega\left(e_{1}^{0}, e_{2}^{0}, e_{3}^{0}\right) .
$$

Proposition 5.5. Let $\left(E_{-1}, E_{0}, \partial, \rho, S, \diamond, \Omega\right)$ be an exact CLWX 2-algebroid. Then $\left(F_{-1} \oplus E_{0} ; \rho, l_{1}\right.$, $\left.l_{2}, l_{3}\right)$ is a quadratic Lie 2-algebroid, which is called the ample Lie 2-algebroid. Consequently, the exact CLWX 2-algebroid $\left(E_{-1}, E_{0}, \partial, \rho, S, \diamond, \Omega\right)$ is an extension, called CLWX-extension, of the quadratic Lie 2-algebroid $\left(F_{-1} \oplus E_{0} ; \rho, l_{1}, l_{2}, l_{3}\right)$ by $T^{*}[1] M$, i.e.

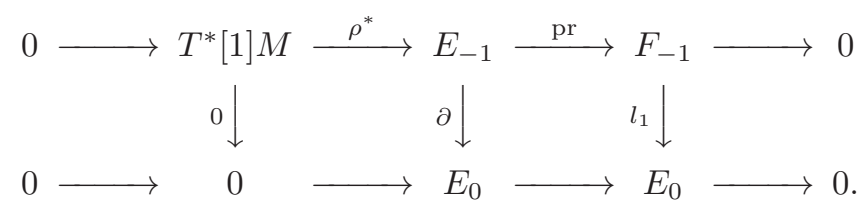


Proof. By the exactness, we can deduce that $l_{1}$ is well-defined. By [13, Lemma 3.7], we can deduce that $l_{2}$ is also well-defined. Since the obstruction of the operation $\diamond$ being skew-symmetric is given in $\rho^{*}\left(T^{*}[1] M\right)$, it follows that $l_{2}$ is skew-symmetric. Since $\partial \circ \rho^{*}=0$, we obtain a Lie 2-algebra $\left(\Gamma\left(F_{-1}\right) \oplus \Gamma\left(E_{0}\right) ; l_{1}, l_{2}, l_{3}\right)$. Therefore, $\left(F_{-1} \oplus E_{0} ; \rho, l_{1}, l_{2}, l_{3}\right)$ is a transitive Lie 2-algebroid. It is obvious that the pairing $S$ vanishes restricting on $\rho^{*}\left(T^{*}[1] M\right) \oplus \operatorname{ker}(\rho)$. Thus, it induces a nondegenerate graded symmetric pairing $\mathcal{S}$ on $F_{-1} \oplus \operatorname{ker}(\rho)$ by

$$
\mathcal{S}(u, m)=S(u, \widetilde{m}), \quad \forall m \in \Gamma\left(F_{-1}\right), \widetilde{m} \in \Gamma\left(E_{-1}\right) \text { such that } \operatorname{pr}(\widetilde{m})=m .
$$

It is straightforward to see that invariant conditions in Definition 4.1 are satisfied. Thus, the transitive Lie 2-algebroid $\left(F_{-1} \oplus E_{0} ; \rho, l_{1}, l_{2}, l_{3}\right)$ is a quadratic Lie 2-algebroid.

Let $\left(A_{-1} \oplus A_{0} ; \rho, l_{1}, l_{2}, l_{3}\right)$ be a quadratic Lie 2-algebroid, in which $l_{1}$ is injective. Now we use the notations as in Subsection 3.1. Denote by $\mathcal{G}=\operatorname{ker}(\rho)$. By the nondegeneracy of $\mathcal{S}$, we have $A_{-1} \cong \mathcal{G}^{*}$ and we will write $A_{-1}=\mathcal{G}^{*}[1]$ directly. Then $\mathcal{S}$ is simply given by (33). The transitive Lie 2 -algebroid $\left(\mathcal{G}^{*}[1] \oplus A_{0} ; \rho, l_{1}, l_{2}, l_{3}\right)$ fits the following exact sequence:

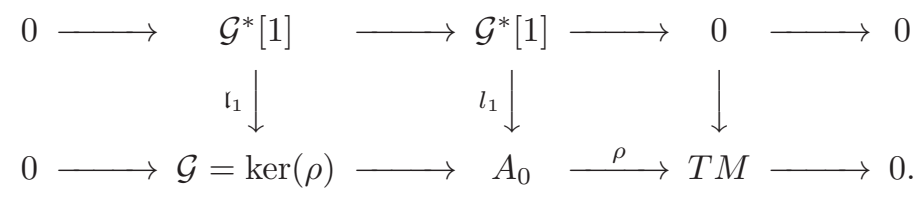

We use $\left(\mathfrak{l}_{1}, \mathfrak{l}_{2}, \mathfrak{l}_{3}\right)$ to denote the graded bundle of quadratic Lie 2-algebra structures on $\mathcal{G}^{*}[1] \oplus \mathcal{G}$. Since $l_{1}$ is injective, $\mathfrak{l}_{1}$ is an isomorphism. Choose a splitting $(\sigma, \gamma)$ of the quadratic Lie 2-algebroid $\left(\mathcal{G}^{*}[1] \oplus A_{0} ; \rho, l_{1}, l_{2}, l_{3}\right)$, then $A_{0} \cong T M \oplus \mathcal{G}$ and $\rho$ is exactly the projection $\mathrm{pr}_{T M}$. Define $\nabla^{0}$ : $\Gamma(T M) \times \Gamma(\mathcal{G}) \longrightarrow \Gamma(\mathcal{G}), \nabla^{1}: \Gamma(T M) \times \Gamma\left(\mathcal{G}^{*}\right) \longrightarrow \Gamma\left(\mathcal{G}^{*}\right)$ and $R \in \Omega^{2}(T M, \mathcal{G})$ by (7) and (8) respectively. Define totally skewsymmetric bundle maps $I: \wedge^{3} T M \longrightarrow \mathcal{G}^{*}, J: \wedge^{2} T M \otimes \mathcal{G} \longrightarrow \mathcal{G}^{*}$ and $K: T M \otimes \wedge^{2} \mathcal{G} \longrightarrow \mathcal{G}^{*}$ by (9)-(11) respectively. Then $I_{\gamma}, J, K$ induce totally skew-symmetric bundle maps $I_{\gamma}^{b}: \wedge^{2} T M \otimes \mathcal{G} \longrightarrow T^{*} M, J^{b}: T M \otimes \wedge^{2} \mathcal{G} \longrightarrow T^{*} M$ and $K^{b}: \wedge^{3} \mathcal{G} \longrightarrow T^{*} M$ by

$$
\begin{aligned}
\left\langle I_{\gamma}^{b}(X, Y, u), Z\right\rangle & =-\left\langle u, I_{\gamma}(X, Y, Z)\right\rangle, \\
\left\langle J^{b}(X, u, v), Y\right\rangle & =-\langle v, J(X, u, Y)\rangle, \\
\left\langle K^{b}(u, v, w), X\right\rangle & =-\langle w, K(X, u, v)\rangle,
\end{aligned}
$$

for all $X, Y, Z \in \mathfrak{X}(M)$ and $u, v, w \in \Gamma(\mathcal{G})$.

Theorem 5.6. Let $\left(A_{-1} \oplus A_{0} ; \rho, l_{1}, l_{2}, l_{3}\right)$ be a quadratic Lie 2-algebroid, in which $l_{1}$ is injective. Then it admits a CLWX-extension if and only if its first Pontryagin class $\left[\mathcal{S}\left(R_{\gamma}, I_{\gamma}\right)\right] \in H^{5}(M)$ is trivial.

Proof. Assume that the quadratic Lie 2-algebroid $\left(A_{-1} \oplus A_{0} ; \rho, l_{1}, l_{2}, l_{3}\right)$ admits a CLWX-extension. Using the same notations as in Section 4 , let $\left(\mathcal{G}^{*} \oplus \mathcal{G} ; \mathfrak{l}_{1}, \mathfrak{l}_{2}, \mathfrak{l}_{3}\right)$ be the corresponding graded bundle of Lie 2-algebras. Let $\left(E_{-1}, E_{0}, \partial, \rho, S, \diamond, \Omega\right)$ be an exact CLWX 2-algebroid whose ample Lie 2algebroid is $\left(A_{-1} \oplus A_{0} ; \rho, l_{1}, l_{2}, l_{3}\right)$. Then we have $E_{0}=A_{0}$. We go on choosing a section $\lambda$ on $\mathcal{G}^{*}[1]$ that is orthogonal to $\sigma$, i.e. $S(\lambda(m), \sigma(X))=0$ for all $m \in \Gamma\left(\mathcal{G}^{*}[1]\right)$ and $X \in \mathfrak{X}(M)$. It turns out that $E_{-1} \cong \rho^{*}\left(T^{*}[1] M\right) \oplus \lambda\left(\mathcal{G}^{*}[1]\right)$. Since $\rho$ is surjective, $\rho^{*}$ is injective, we deduce that $E_{-1} \cong T^{*}[1] M \oplus \mathcal{G}^{*}[1]$ and the nondegenerate bilinear form is exactly given by

$$
S(X+u, \alpha+m)=\langle\alpha, X\rangle+\langle m, u\rangle, \quad \forall X \in \mathfrak{X}(M), \alpha \in \Omega^{1}(M), u \in \Gamma(\mathcal{G}), m \in \Gamma\left(\mathcal{G}^{*}\right) .
$$


Define $H \in \Omega^{4}(M)$ by

$$
H(X, Y, Z, W)=S(\Omega(\sigma(X), \sigma(Y), \sigma(Z)), \sigma(W)) .
$$

Transfer the CLWX 2-algebroid structure to $\left(T^{*}[1] M \oplus \mathcal{G}^{*}[1]\right) \oplus(T M \oplus \mathcal{G})$, we have

$$
\left\{\begin{aligned}
\partial(\alpha+m)= & \mathfrak{l}_{1}(m), \\
(X+u) \diamond(Y+v)= & {[X, Y]+\nabla_{X}^{0} v-\nabla_{Y}^{0} u-R_{\gamma}(X, Y)+\mathfrak{l}_{2}(u, v), } \\
(X+u) \diamond(\alpha+m)= & L_{X} \alpha+\nabla_{X}^{1} m+Q(X, m)+\mathfrak{l}_{2}(u, m)+P_{1}(u, m), \\
(\alpha+m) \diamond(X+u)= & -\iota_{X} d \alpha-\nabla_{X}^{1} m-Q(X, m)+\mathfrak{l}_{2}(m, u)+P_{2}(m, u), \\
\Omega(X+u, Y+v, Z+w)= & H(X, Y, Z)-I_{\gamma}^{\mathrm{b}}(X, Y, w)-I_{\gamma}^{\mathrm{b}}(X, v, Z)-I_{\gamma}^{\mathrm{b}}(u, Y, Z) \\
& -J^{\mathrm{b}}(X, v, w)-J^{\mathrm{b}}(u, Y, w)-J^{\mathrm{b}}(u, v, Z)+K^{\mathrm{b}}(u, v, w) \\
& -I_{\gamma}(X, Y, Z)-J(X, Y, w)-J(X, v, Z)-J(u, Y, Z) \\
& +K(X, v, w)+K(u, Y, w)+K(u, v, Z)+\mathfrak{l}_{3}(u, v, w),
\end{aligned}\right.
$$

where $Q: \mathfrak{X}(M) \times \Gamma\left(\mathcal{G}^{*}\right) \longrightarrow \Omega^{1}(M)$ is defined by

$$
\langle Q(X, m), Y\rangle=\left\langle m, R_{\gamma}(X, Y)\right\rangle,
$$

and $P_{1}: \Gamma(\mathcal{G}) \times \Gamma\left(\mathcal{G}^{*}\right) \longrightarrow \Omega^{1}(M)$ and $P_{2}: \Gamma\left(\mathcal{G}^{*}\right) \times \Gamma(\mathcal{G}) \longrightarrow \Omega^{1}(M)$ are defined by

$$
\left\langle P_{1}(u, m), X\right\rangle=\left\langle m, \nabla_{X}^{0} u\right\rangle, \quad\left\langle P_{2}(m, u), X\right\rangle=\left\langle u, \nabla_{X}^{1} m\right\rangle .
$$

Then by the Jacobiator identity that $\Omega$ should satisfy, for all $W, X, Y, Z \in \mathfrak{X}(M)$, we have

$$
\begin{aligned}
& W \diamond \Omega(X, Y, Z)-X \diamond \Omega(W, Y, Z)+Y \diamond \Omega(W, X, Z)+\Omega(W, X, Y) \diamond Z \\
& -\Omega(W \diamond X, Y, Z)+\Omega(W \diamond Y, X, Z)-\Omega(W \diamond Z, X, Y) \\
& -\Omega(X \diamond Y, W, Z)+\Omega(X \diamond Z, W, Y)-\Omega(Y \diamond Z, W, X) \\
= & W \diamond(H(X, Y, Z)-I(X, Y, Z))-X \diamond(H(W, Y, Z)-I(W, Y, Z)) \\
& +Y \diamond(H(W, X, Z)-I(W, X, Z))+(H(W, X, Y)-I(W, X, Y)) \diamond Z \\
& -\Omega\left([W, X]-R_{\gamma}(W, X), Y, Z\right)+\Omega\left([W, Y]-R_{\gamma}(W, Y), X, Z\right)-\Omega\left([W, Z]-R_{\gamma}(W, Z), X, Y\right) \\
& -\Omega\left([X, Y]-R_{\gamma}(X, Y), W, Z\right)+\Omega\left([X, Z]-R_{\gamma}(X, Z), W, Y\right)-\Omega\left([Y, Z]-R_{\gamma}(Y, Z), W, X\right) \\
= & L_{W} H(X, Y, Z)-L_{X} H(W, Y, Z)+L_{Y} H(W, X, Z)-\iota_{Z} d(H(W, X, Y)) \\
& -H([W, X], Y, Z)+H([W, Y], X, Z)-H([W, Z], X, Y) \\
& -H([X, Y], W, Z)+H([X, Z], W, Y)-H([Y, Z], W, X) \\
& -\nabla_{W}^{1} I_{\gamma}(X, Y, Z)-Q\left(W, I_{\gamma}(X, Y, Z)\right)+\nabla_{X}^{1} I_{\gamma}(W, Y, Z)+Q\left(X, I_{\gamma}(W, Y, Z)\right) \\
& -\nabla_{Y}^{1} I_{\gamma}(W, X, Z)-Q\left(Y, I_{\gamma}(W, X, Z)\right)+\nabla_{Z}^{1} I_{\gamma}(W, X, Y)+Q\left(Z, I_{\gamma}(W, X, Y)\right) \\
& +I_{\gamma}([W, X], Y, Z)-I_{\gamma}([W, Y], X, Z)+I_{\gamma}([W, Z], X, Y) \\
& +I_{\gamma}([X, Y], W, Z)-I_{\gamma}([X, Z], W, Y)+I_{\gamma}([Y, Z], W, X) \\
& -I_{\gamma}^{b}\left(R_{\gamma}(W, X), Y, Z\right)+I_{\gamma}^{b}\left(R_{\gamma}(W, Y), X, Z\right)-I_{\gamma}^{b}\left(R_{\gamma}(W, Z), X, Y\right) \\
& -I_{\gamma}^{b}\left(R_{\gamma}(X, Y), W, Z\right)+I_{\gamma}^{b}\left(R_{\gamma}(X, Z), W, Y\right)-I_{\gamma}^{b}\left(R_{\gamma}(Y, Z), W, X\right) \\
& -J\left(R_{\gamma}(W, X), Y, Z\right)+J\left(R_{\gamma}(W, Y), X, Z\right)-J\left(R_{\gamma}(W, Z), X, Y\right) \\
& -J\left(R_{\gamma}(X, Y), W, Z\right)+J\left(R_{\gamma}(X, Z), W, Y\right)-J\left(R_{\gamma}(Y, Z), W, X\right) \\
= & d H(W, X, Y, Z, \cdot)+\mathcal{S}\left(R_{\gamma}, I_{\gamma}\right)(W, X, Y, Z, \cdot)-\left(d_{\nabla} I_{\gamma}+J \circ R_{\gamma}\right)(W, X, Y, Z) \\
= & 0
\end{aligned}
$$


In particular, we have $\mathcal{S}\left(R_{\gamma}, I_{\gamma}\right)+d H=0$, which implies that the first Pontryagin class $\left[\mathcal{S}\left(R_{\gamma}, I_{\gamma}\right)\right] \in$ $H^{5}(M)$ is trivial.

Conversely, if the first Pontryagin class is trivial, then there exists a 4 -form $H \in \Omega^{4}(M)$ such that

$$
\mathcal{S}\left(R_{\gamma}, I_{\gamma}\right)+d H=0 .
$$

On the graded bundle $\left(T^{*}[1] M \oplus \mathcal{G}^{*}[1]\right) \oplus(T M \oplus \mathcal{G})$, define $\partial, \diamond, \Omega$ by (64). We are going to show that $\left(\left(T^{*}[1] M \oplus \mathcal{G}^{*}[1]\right) \oplus(T M \oplus \mathcal{G}), \partial, \operatorname{pr}_{T M}, S, \diamond, \Omega\right)$ is a CLWX 2-algebroid. First we show that under conditions in Theorem 3.3 and by Proposition 4.2 and [67]), $\left(\Omega^{1}(M) \oplus \Gamma\left(\mathcal{G}^{*}\right), \mathfrak{X}(M) \oplus \Gamma(\mathcal{G}), \partial, \diamond, \Omega\right)$ is a Leibniz 2-algebra. This is the most intrinsic part in the proof. By (13) and (34), we can deduce that Axioms (a), (b), (c) in Definition 2.1 hold by straightforward computations. Note that the restriction of $\diamond$ on $\wedge^{2} \Gamma(T M \oplus \mathcal{G})$ is the same as the one for the transitive Lie 2-algebroid given in (12), we deduce that Axiom (d) in Definition 2.1 holds by the fact $\left.\partial\right|_{\Omega^{1}(M)}=0$. By straightforward computation, we have

$$
\begin{aligned}
& (X+u) \diamond((Y+v) \diamond(\alpha+m))-((X+u) \diamond(Y+v)) \diamond(\alpha+m) \\
& -(Y+v) \diamond((X+u) \diamond(\alpha+m)) \\
= & l_{2}\left(X+u, l_{2}(Y+v, m)\right)-l_{2}\left(l_{2}(X+u, Y+v), m\right)-l_{2}\left(Y+v, l_{2}(X+u, m)\right) \\
& +L_{X}\left(L_{Y} \alpha+Q(Y, m)+P_{1}(v, m)\right)+Q\left(X, \nabla_{Y}^{1} m+\mathfrak{l}_{2}(v, m)\right)+P_{1}\left(u, \nabla_{Y}^{1} m+\mathfrak{l}_{2}(v, m)\right) \\
& -L_{[X, Y]} \alpha-Q([X, Y], m)-P_{1}\left(\nabla_{X}^{0} v-\nabla_{Y}^{0} u-R_{\gamma}(X, Y)+\mathfrak{l}_{2}(u, v), m\right) \\
& -L_{Y}\left(L_{X} \alpha+Q(X, m)+P_{1}(u, m)\right)-Q\left(Y, \nabla_{X}^{1} m+\mathfrak{l}_{2}(u, m)\right)-P_{1}\left(v, \nabla_{X}^{1} m+\mathfrak{l}_{2}(u, m)\right),
\end{aligned}
$$

where $l_{2}$ is given by (12). By Theorem 3.3, we have

$$
\begin{aligned}
& l_{2}\left(X+u, l_{2}(Y+v, m)\right)-l_{2}\left(l_{2}(X+u, Y+v), m\right)-l_{2}\left(Y+v, l_{2}(X+u, m)\right) \\
= & l_{3}\left(X+u, Y+v, \mathfrak{l}_{1}(m)\right) \\
= & -J\left(X, Y, \mathfrak{l}_{1}(m)\right)+K\left(X, v, \mathfrak{l}_{1}(m)\right)+K\left(u, Y, \mathfrak{l}_{1}(m)\right)+\mathfrak{l}_{3}\left(u, v, \mathfrak{l}_{1}(m)\right) .
\end{aligned}
$$

Thus, Axiom $\left(e_{1}\right)$ in Definition 2.1 holds if and only if

$$
\begin{aligned}
& L_{X}\left(Q(Y, m)+P_{1}(v, m)\right)+Q\left(X, \nabla_{Y}^{1} m+\mathfrak{l}_{2}(v, m)\right)+P_{1}\left(u, \nabla_{Y}^{1} m+\mathfrak{l}_{2}(v, m)\right) \\
& -Q([X, Y], m)-P_{1}\left(\nabla_{X}^{0} v-\nabla_{Y}^{0} u-R_{\gamma}(X, Y)+\mathfrak{l}_{2}(u, v), m\right) \\
& -L_{Y}\left(Q(X, m)+P_{1}(u, m)\right)-Q\left(Y, \nabla_{X}^{1} m+\mathfrak{l}_{2}(u, m)\right)-P_{1}\left(v, \nabla_{X}^{1} m+\mathfrak{l}_{2}(u, m)\right) \\
= & -I_{\gamma}^{b}\left(X, Y, \mathfrak{l}_{1}(m)\right)-J^{b}\left(X, v, \mathfrak{l}_{1}(m)\right)-J^{b}\left(u, Y, \mathfrak{l}_{1}(m)\right)+K^{b}\left(u, v, \mathfrak{l}_{1}(m)\right),
\end{aligned}
$$

which can be obtained by (34), (35), (19), (14), (37) and (17). We omit the details.

Similarly, we can show that Axiom $\left(e_{2}\right)$ and $\left(e_{3}\right)$ in Definition 2.1 hold.

The last step to show that $\left(\left(\Omega^{1}(M) \oplus \Gamma\left(\mathcal{G}^{*}\right)\right) \oplus(\mathfrak{X}(M) \oplus \Gamma(\mathcal{G})) ; \partial, \diamond, \Omega\right)$ is a Leibniz 2-algebra is to show the Jacobiator identity for $\Omega$. Roughly speaking, the Jacobiator identity for $\Omega$ is due to (16), (20), (21), (23) and (67). We leave the proof to readers. This finishes the proof of $\left(\left(\Omega^{1}(M) \oplus \Gamma\left(\mathcal{G}^{*}\right)\right) \oplus(\mathfrak{X}(M) \oplus \Gamma(\mathcal{G})) ; \partial, \diamond, \Omega\right)$ being a Leibniz 2-algebra.

By (37), we have

$$
\begin{aligned}
(X+u) \diamond(\alpha+m)+(\alpha+m) \diamond(X+u) & =L_{X} \alpha-\iota_{X} d \alpha+P_{1}(u, m)+P_{2}(m, u) \\
& =d(\langle X, \alpha\rangle+\langle u, m\rangle) \\
& =d S(X+u, \alpha+m),
\end{aligned}
$$


which implies that Axiom (ii) in Definition 2.5 holds.

Finally, by Proposition 4.2, we can deduce that Axioms (iii)-(iv) in Definition 2.5 hold directly. The proof is finished.

\section{The first Pontryagin class of a trivial principle 2-bundle with a $\Gamma$-connection}

\subsection{Strict Lie 2-groups and strict Lie 2-algebras}

A group is a monoid where every element has an inverse. A 2-group is a monoidal category where every object has a weak inverse and every morphism has an inverse. Denote the category of smooth manifolds and smooth maps by Diff, a (semistrict) Lie 2-group is a 2-group in DiffCat, where DiffCat is the 2-category consisting of categories, functors, and natural transformations in Diff. For more details, see [3, 4]. Here we only give the definition of a strict Lie 2-group.

Definition 6.1. A strict Lie 2-group is a Lie groupoid $C$ such that

(a) The space of morphisms $C_{1}$ and the space of objects $C_{0}$ are Lie groups.

(b) The source and the target $s, t: C_{1} \longrightarrow C_{0}$, the identity assigning function $i: C_{0} \longrightarrow C_{1}$ and the composition $\circ: C_{1} \times{ }_{C_{0}} C_{1} \longrightarrow C_{1}$ are all Lie group morphisms.

In the following we will denote the composition $\circ$ in the Lie groupoid structure by $\cdot_{\mathrm{v}}: C_{1} \times{ }_{C_{0}}$ $C_{1} \longrightarrow C_{1}$ and call it the vertical multiplication. Denote the Lie 2-group multiplication by ${ }^{\mathrm{h}}$ : $C \times C \longrightarrow C$ and call it the horizontal multiplication.

It is well known that strict Lie 2-groups can be described by crossed modules of Lie groups.

Definition 6.2. A crossed module of Lie groups is a quadruple $\left(H_{1}, H_{0}, \Psi, \Phi\right)$, which we denote simply by $\mathbb{H}$, where $H_{1}$ and $H_{0}$ are Lie groups, $\Psi: H_{1} \longrightarrow H_{0}$ is a Lie group morphism, and $\Phi: H_{0} \times H_{1} \longrightarrow H_{1}$ is an action of $H_{0}$ on $H_{1}$ preserving the Lie group structure of $H_{1}$ such that the Lie group morphism $\Psi$ is $H_{0}$-equivariant:

$$
\Psi\left(\Phi_{g}(h)\right)=g \Psi(h) g^{-1}, \quad \forall g \in H_{0}, h \in H_{1},
$$

and $\Psi$ satisfies the so called Perffer identity:

$$
\Phi_{\Psi(h)}\left(h^{\prime}\right)=h h^{\prime} h^{-1}, \quad \forall h, h^{\prime} \in H_{1} .
$$

Theorem 6.3. There is a one-to-one correspondence between crossed modules of Lie groups and strict Lie 2-groups.

Roughly speaking, given a crossed module $\left(H_{1}, H_{0}, \Psi, \Phi\right)$ of Lie groups, there is a strict Lie 2-group for which $C_{0}=H_{0}$ and $C_{1}=H_{0} \ltimes H_{1}$, the semidirect product of $H_{0}$ and $H_{1}$. In this strict Lie 2-group, the source and target maps $s, t: C_{1} \longrightarrow C_{0}$ are given by

$$
s(g, h)=g, \quad t(g, h)=t(h) \cdot g,
$$

the vertical multiplication $\cdot_{\mathrm{v}}$ is given by:

$$
\left(g^{\prime}, h^{\prime}\right) \cdot{ }_{\mathrm{v}}(g, h)=\left(g, h^{\prime} \cdot h\right), \quad \text { where } g^{\prime}=t(h) \cdot g,
$$

the horizontal multiplication $\cdot h$ is given by

$$
(g, h) \cdot_{\mathrm{h}}\left(g^{\prime}, h^{\prime}\right)=\left(g \cdot g^{\prime}, h \cdot \Phi_{g} h^{\prime}\right) .
$$


Definition 6.4. A crossed module of Lie algebras is a quadruple $\left(\mathfrak{h}_{1}, \mathfrak{h}_{0}, \psi, \phi\right)$, which we denote by $\mathbb{h}$, where $\mathfrak{h}_{1}$ and $\mathfrak{h}_{0}$ are Lie algebras, $\psi: \mathfrak{h}_{1} \longrightarrow \mathfrak{h}_{0}$ is a Lie algebra morphism and $\phi: \mathfrak{h}_{0} \longrightarrow \operatorname{Der}\left(\mathfrak{h}_{1}\right)$ is an action of Lie algebra $\mathfrak{h}_{0}$ on Lie algebra $\mathfrak{h}_{1}$ as a derivation, such that

$$
\psi\left(\phi_{u}(m)\right)=[u, \psi(m)]_{\mathfrak{h}_{0}}, \quad \phi_{\psi(m)}(p)=[m, p]_{\mathfrak{h}_{1}}, \quad \forall u \in \mathfrak{h}_{0}, m, p \in \mathfrak{h}_{1} .
$$

Example 6.5. For any Lie algebra $\mathfrak{k}$, the adjoint action ad is a Lie algebra morphism from $\mathfrak{k}$ to $\operatorname{Der}(\mathfrak{k})$. Then $(\mathfrak{k}, \operatorname{Der}(\mathfrak{k})$, ad, id) is a crossed module of Lie algebras.

Theorem 6.6. There is a one-to-one correspondence between 2-term DGLAs (strict Lie 2-algebras) and crossed modules of Lie algebras.

In short, the formula for the correspondence can be given as follows: A 2-term DGLA $\left(V_{-1} \oplus\right.$ $\left.V_{0} ; \mathfrak{l}_{1}, \mathfrak{l}_{2}\right)$ gives rise to a Lie algebra crossed module with $\mathfrak{h}_{1}=V_{-1}$ and $\mathfrak{h}_{0}=V_{0}$, where the Lie brackets are given by:

$$
[m, p]_{\mathfrak{h}_{1}}=\mathfrak{l}_{2}\left(\mathfrak{l}_{1}(m), p\right), \quad[u, v]_{\mathfrak{h}_{0}}=\mathfrak{l}_{2}(u, v), \quad \forall m, p \in V_{-1}, u, v \in V_{0},
$$

and $\psi=\mathfrak{l}_{1}, \phi: \mathfrak{h}_{0} \longrightarrow \operatorname{Der}\left(\mathfrak{h}_{1}\right)$ is given by $\phi_{u}(m)=\mathfrak{l}_{2}(u, m)$. The DGLA structure gives the Jacobi identity for $[\cdot, \cdot]_{\mathfrak{h}_{1}}$ and $[\cdot, \cdot]_{\mathfrak{h}_{0}}$, and various other conditions for crossed modules.

Conversely, a crossed module $\left(\mathfrak{h}_{1}, \mathfrak{h}_{0}, \psi, \phi\right)$ gives rise to a 2-term DGLA with $V_{-1}=\mathfrak{h}_{1}, V_{0}=\mathfrak{h}_{0}$, $\mathfrak{l}_{1}=\psi$, and $\mathfrak{l}_{2}$ given by:

$$
\mathfrak{l}_{2}(u, v) \triangleq[u, v]_{\mathfrak{h}_{0}}, \quad \mathfrak{l}_{2}(u, m) \triangleq \phi_{u}(m), \quad \forall u, v \in \mathfrak{h}_{0}, m \in \mathfrak{h}_{1} .
$$

\subsection{The transitive Lie 2-algebroid associated to a trivial principle 2- bundle with a $\Gamma$-connection}

First we review the notion of a principle 2-bundle for a strict Lie 2-group $\Gamma$ on the basis of [28, 30]. Let $\Gamma$ be a strict Lie 2 -group corresponding to the Lie group crossed module $\left(H_{1}, H_{0}, \Psi, \Phi\right)$. Let $\left(\mathfrak{h}_{1}, \mathfrak{h}_{0}, \psi, \phi\right)$ be the crossed module of Lie algebras corresponding to $\left(H_{1}, H_{0}, \Psi, \Phi\right)$, and $\left(\mathfrak{h}_{1} \oplus\right.$ $\left.\mathfrak{h}_{0} ; \mathfrak{l}_{1}, \mathfrak{l}_{2}\right)$ the associated strict Lie 2-algebra.

Definition 6.7. A principle $\Gamma$-2-bundle over a differential manifold $M$ is a Lie groupoid $\mathcal{P}$, a surjective submersion functor $\pi: \mathcal{P} \longrightarrow M_{\mathrm{dis}}$, and a smooth right action $R$ of $\Gamma$ on $\mathcal{P}$ that preserves $\pi$, such that the smooth functor

$$
\left(\mathrm{pr}_{1}, R\right): \mathcal{P} \times \Gamma \longrightarrow \mathcal{P} \times_{M} \mathcal{P}
$$

is a weak equivalence, where $M_{\mathrm{dis}}$ is the Lie groupoid with objects $M$ and only identity morphisms.

It is obvious that $M_{\mathrm{dis}} \times \Gamma$ is a principle $\Gamma$-2-bundle, which is called the trivial principle $\Gamma$-2bundle.

A $\Gamma$-connection on $M$ is a pair $(A, B)$ consisting of an $\mathfrak{h}_{0^{-}}$-valued 1 -form $A \in \Omega^{1}\left(M, \mathfrak{h}_{0}\right)$ and an $\mathfrak{h}_{1}$-valued 2 -form $B \in \Omega^{2}\left(M, \mathfrak{h}_{1}\right)$. The curvature curv and the fake-curvature fcurv of a $\Gamma$-connection $(A, B)$ are defined by

$$
\begin{aligned}
\operatorname{curv}(A, B) & \triangleq d B+\mathfrak{l}_{2}(A, B) \in \Omega^{3}\left(M, \mathfrak{h}_{1}\right) \\
\operatorname{fcurv}(A, B) & \triangleq d A+\frac{1}{2} \mathfrak{l}_{2}(A, A)-\mathfrak{l}_{1}(B) \in \Omega^{2}\left(M, \mathfrak{h}_{0}\right)
\end{aligned}
$$


Every $\Gamma$-connection can give rise to a connection on the trivial principle $\Gamma$-2-bundle $M_{\text {dis }} \times \Gamma$. See [28, Lemma 5.4.1] for more details.

Given a $\Gamma$-connection $(A, B)$, we can construct a transitive Lie 2 -algebroid, which can be viewed as the infinitesimal of the trivial principle $\Gamma$-2-bundle with a connection, as follows: let $A_{-1}=$ $M \times \mathfrak{h}_{1}, A_{0}=T M \oplus\left(M \times \mathfrak{h}_{0}\right)$, and define $l_{1}, l_{2}, l_{3}$ by

$$
\left\{\begin{aligned}
l_{1}(m)= & \mathfrak{l}_{1}(m), \\
l_{2}(X+u, Y+v)= & {[X, Y]+L_{X} v+\mathfrak{l}_{2}(A(X), v)-L_{Y} u-\mathfrak{l}_{2}(A(Y), u) } \\
& +\operatorname{fcurv}(A, B)(X, Y)+\mathfrak{l}_{2}(u, v), \\
l_{2}(X+u, m)= & L_{X} m+\mathfrak{l}_{2}(A(X), m)+\mathfrak{l}_{2}(u, m), \\
l_{3}(X+u, Y+v, Z+w)= & -\operatorname{curv}(A, B)(X, Y, Z)+\mathfrak{l}_{2}(B(X, Y), w) \\
& +\mathfrak{l}_{2}(B(Y, Z), u)+\mathfrak{l}_{2}(B(Z, X), v),
\end{aligned}\right.
$$

for all $X, Y, Z \in \mathfrak{X}(M), u, v, w \in \Gamma\left(M \times \mathfrak{h}_{0}\right)$ and $m \in \Gamma\left(M \times \mathfrak{h}_{1}\right)$.

Theorem 6.8. Let $(A, B)$ be a $\Gamma$-connection on $M$. Then $\left(\left(M \times \mathfrak{h}_{1}\right) \oplus\left(T M \oplus\left(M \times \mathfrak{h}_{0}\right)\right) ; \rho=\right.$ $\left.\mathrm{pr}_{T M}, l_{1}, l_{2}, l_{3}\right)$ is a transitive Lie 2-algebroid, where $l_{i}$ are given by (172).

Proof. Compare to (12), we write

$$
\begin{aligned}
\nabla_{X}^{0} u & =L_{X} u+\mathfrak{l}_{2}(A(X), u), \\
\nabla_{X}^{1} m & =L_{X} m+\mathfrak{l}_{2}(A(X), m), \\
R & =-d A-\frac{1}{2} \mathfrak{l}_{2}(A, A), \\
\gamma & =B, \\
J(X, Y, w) & =-\mathfrak{l}_{2}(B(X, Y), w),
\end{aligned}
$$

and $I=0, K=0, \mathfrak{l}_{3}=0$. Now we have

$$
I_{\gamma}=I+d_{\nabla^{1}} \gamma=d_{\nabla^{1}} B=d B+\mathfrak{l}_{2}(A, B)=\operatorname{curv}(A, B) .
$$

Then $\left(\left(M \times \mathfrak{h}_{1}\right) \oplus\left(T M \oplus\left(M \times \mathfrak{h}_{0}\right)\right) ; \rho=\operatorname{pr}_{T M}, l_{1}, l_{2}, l_{3}\right)$ is a transitive Lie 2-algebroid if and only if (13)-21) hold. Since $l_{1}$ is $C^{\infty}(M)$-linear, (13) holds naturally. Since $K=0$ and $\mathfrak{l}_{3}=0$, we can deduce that (14)-(16) hold. It is also straightforward to deduce that (17) and (18) hold. By $d \mathfrak{l}_{2}(A, A)=2 \mathfrak{l}_{2}(d A, A)$ and the Jacobi identity for $\mathfrak{l}_{2}$, we have

$$
\begin{aligned}
& \nabla_{X}^{0} R(Y, Z)-R([X, Y], Z)+c . p . \\
= & L_{X} R(Y, Z)-R([X, Y], Z)+c \cdot p \cdot+\mathfrak{l}_{2}(A(X), R(Y, Z))+c . p . \\
= & d R(X, Y, Z)+\mathfrak{l}_{2}(A, R)(X, Y, Z) \\
= & d\left(-d A-\frac{1}{2} \mathfrak{l}_{2}(A, A)\right)(X, Y, Z)+\mathfrak{l}_{2}\left(A,-d A-\frac{1}{2} \mathfrak{l}_{2}(A, A)\right)(X, Y, Z) \\
= & 0,
\end{aligned}
$$

which implies that (19) holds. It is straightforward to deduce that (20) holds. Finally, by $d \mathfrak{l}_{2}(A, \gamma)=\mathfrak{l}_{2}(d A, \gamma)-\mathfrak{l}_{2}(A, d \gamma)$ and $\mathfrak{l}_{2}\left(\mathfrak{l}_{1}(m), p\right)=\mathfrak{l}_{2}\left(m, \mathfrak{l}_{1}(p)\right)$, we have

$$
\begin{aligned}
d_{\nabla^{1}}\left(I+d_{\nabla^{1}} \gamma\right) & =d_{\nabla^{1}}\left(d_{\nabla^{1}} \gamma\right) \\
& =d\left(d_{\nabla^{1}} \gamma\right)+\mathfrak{l}_{2}\left(A, d_{\nabla^{1}} \gamma\right) \\
& =d\left(d \gamma+\mathfrak{l}_{2}(A, \gamma)\right)+\mathfrak{l}_{2}\left(A, d \gamma+\mathfrak{l}_{2}(A, \gamma)\right) \\
& =\mathfrak{l}_{2}(d A, \gamma)+\mathfrak{l}_{2}\left(A, \mathfrak{l}_{2}(A, \gamma)\right) \\
& =\mathfrak{l}_{2}\left(d A+\frac{1}{2} \mathfrak{l}_{2}(A, A), \gamma\right),
\end{aligned}
$$


and

$$
J \circ R_{\gamma}=-\mathfrak{l}_{2}\left(\gamma, R_{\gamma}\right)=-\mathfrak{l}_{2}\left(\gamma, R+\mathfrak{l}_{1} \circ \gamma\right)=\mathfrak{l}_{2}(R, \gamma),
$$

which implies that (21) holds. Thus, $\left(\left(M \times \mathfrak{h}_{1}\right) \oplus\left(T M \oplus\left(M \times \mathfrak{h}_{0}\right)\right) ; \rho=\operatorname{pr}_{T M}, l_{1}, l_{2}, l_{3}\right)$ is a transitive Lie 2-algebroid.

Remark 6.9. As proposed by the referee, it is natural to consider the case of nontrivial principle 2bundle. It is known that principle 2-bundles are classified by the nonabelian differential cohomology. See [19, 29, 30] for more details. A nonabelian cocycle is represented by an open cover $\left\{U_{i}\right\}$ of $M$, together with a collection of smooth maps $g_{i j}: U_{i j} \longrightarrow H_{0}$ and $a_{i j k}: U_{i j k} \longrightarrow H_{1}$ such that $g_{i k}=\Psi\left(a_{i j k}\right) g_{i j} g_{j k}$ and $a_{i k l} \Phi_{g_{k l}} a_{i j k}=a_{i j l} a_{j k l}$. One can see that $g_{i j}$ does not satisfy the cocycle condition. Thus, the naive idea of gluing the local standard model given in Theorem 6.8 to obtain a global object does not work. We will study this interesting question in the future.

\subsection{The first Pontryagin class of the quadratic Lie 2-algebroid associated to a $\Gamma$-connection}

In this subsection, we show that the first Pontryagin class of the quadratic Lie 2-algebroid associated to an $\Gamma$-connection is trivial. First we give an interesting example of quadratic strict Lie 2 -algebras.

Example 6.10. Let $\left(\mathfrak{g},[\cdot, \cdot]_{\mathfrak{g}}, K\right)$ be a quadratic Lie algebra. That is, $K$ is a symmetric nondegenerate bilinear form on $\mathfrak{g}$. Denote by $K^{\sharp}$ the induced map from $\mathfrak{g}$ to $\mathfrak{g}^{*}$, i.e.

$$
K^{\sharp}(u)(v)=K(u, v), \quad \forall u, v \in \mathfrak{g} .
$$

Then $K^{\sharp}$ is an isomorphism. On the graded vector space $\mathfrak{g}^{*}[1] \oplus \mathfrak{g}$, define $\mathfrak{l}_{1}$ and $\mathfrak{l}_{2}$ by

$$
\begin{aligned}
\mathfrak{l}_{1} & =\left(K^{\sharp}\right)^{-1}, \\
\mathfrak{l}_{2}(u+\xi, v+\eta) & =[u, v]_{\mathfrak{g}}+\operatorname{ad}_{u}^{*} \eta-\operatorname{ad}_{v}^{*} \xi, \quad \forall u, v \in \mathfrak{g}, \xi, \eta \in \mathfrak{g}^{*} .
\end{aligned}
$$

Then $\left(\mathfrak{g}^{*}[1] \oplus \mathfrak{g} ; \mathfrak{l}_{1}, \mathfrak{l}_{2}, \mathcal{S}\right)$ is a quadratic strict Lie 2 -algebra, where $\mathcal{S}$ is given by

$$
\mathcal{S}(u+\xi, v+\eta)=\langle\xi, v\rangle+\langle\eta, u\rangle .
$$

In fact, the only nontrivial part of proving $\left(\mathfrak{g}^{*}[1] \oplus \mathfrak{g} ; \mathfrak{l}_{1}, \mathfrak{l}_{2}\right)$ to be a strict Lie 2-algebra is to show the equality

$$
\mathfrak{l}_{2}\left(\left(K^{\sharp}\right)^{-1}(\xi), \eta\right)=\mathfrak{l}_{2}\left(\xi,\left(K^{\sharp}\right)^{-1}(\eta)\right), \quad\left(K^{\sharp}\right)^{-1} \mathfrak{l}_{2}(u, \eta)=\mathfrak{l}_{2}\left(u,\left(K^{\sharp}\right)^{-1}(\eta)\right) .
$$

Since $K^{\sharp}$ is an isomorphism, we can assume that $\xi=K^{\sharp}(u)$ and $\eta=K^{\sharp}(v)$. Since $K$ is invariant, we have

$$
\begin{aligned}
\left\langle\mathfrak{l}_{2}\left(\left(K^{\sharp}\right)^{-1}(\xi), \eta\right)-\mathfrak{l}_{2}\left(\xi,\left(K^{\sharp}\right)^{-1}(\eta)\right), w\right\rangle & =\left\langle\mathfrak{l}_{2}\left(u, K^{\sharp}(v)\right)-\mathfrak{l}_{2}\left(K^{\sharp}(u), v\right), w\right\rangle \\
& =\left\langle\operatorname{ad}_{u}^{*} K^{\sharp}(v)+\operatorname{ad}_{v}^{*} K^{\sharp}(u), w\right\rangle \\
& =-K\left(v,[u, w]_{\mathfrak{g}}\right)-K\left(u,[v, w]_{\mathfrak{g}}\right) \\
& =0 .
\end{aligned}
$$

Similarly, for any $\gamma=K^{\sharp}(w)$, we have

$$
\begin{aligned}
\left\langle\left(K^{\sharp}\right)^{-1} \mathfrak{l}_{2}(u, \eta)-\mathfrak{l}_{2}\left(u,\left(K^{\sharp}\right)^{-1}(\eta)\right), \gamma\right\rangle & =\left\langle\mathfrak{l}_{2}(u, \eta), w\right\rangle-\left\langle\mathfrak{l}_{2}(u, v), K^{\sharp}(w)\right\rangle \\
& =-K\left(v,[u, w]_{\mathfrak{g}}\right)-K\left(w,[u, v]_{\mathfrak{g}}\right) \\
& =0 .
\end{aligned}
$$


Thus, $\left(\mathfrak{g}^{*}[1] \oplus \mathfrak{g} ; \mathfrak{l}_{1}, \mathfrak{l}_{2}\right)$ is a strict Lie 2 -algebra. It is obvious that $\mathcal{S}$ is invariant. Therefore, $\left(\mathfrak{g}^{*}[1] \oplus \mathfrak{g} ; \mathfrak{l}_{1}, \mathfrak{l}_{2}, \mathcal{S}\right)$ is a quadratic strict Lie 2 -algebra.

Now let $\Gamma$ be a strict Lie 2-group such that the corresponding strict Lie 2-algebra is a quadratic strict Lie 2-algebra $\left(\mathfrak{h}^{*}[1] \oplus \mathfrak{h} ; \mathfrak{l}_{1}, \mathfrak{l}_{2}, \mathcal{S}\right)$. Let $(A, B)$ be a $\Gamma$-connection on $M$. Then the transitive Lie 2-algebroid given in Theorem 6.8 is a quadratic Lie 2-algebroid naturally. Consider its first Pontryagin class, we have

Theorem 6.11. Let $\Gamma$ be a strict Lie 2-group such that the corresponding strict Lie 2-algebra is a quadratic strict Lie 2-algebra, and $(A, B)$ a $\Gamma$-connection on $M$. Then the first Pontryagin class associated to the quadratic Lie 2-algebroid given in Theorem 6.8, which is represented by the 5 -cocycle $-\mathcal{S}(\operatorname{fcurv}(A, B), \operatorname{curv}(A, B))$, is trivial.

Proof. First we have

$$
\begin{aligned}
\mathcal{S}(\operatorname{fcurv}(A, B), \operatorname{curv}(A, B))= & \mathcal{S}\left(d A+\frac{1}{2} \mathfrak{l}_{2}(A, A)-\mathfrak{l}_{1}(B), d B+\mathfrak{l}_{2}(A, B)\right) \\
= & \mathcal{S}(d A, d B)+\mathcal{S}\left(d A, \mathfrak{l}_{2}(A, B)\right)+\mathcal{S}\left(\frac{1}{2} \mathfrak{l}_{2}(A, A), d B\right) \\
& +\mathcal{S}\left(\frac{1}{2} \mathfrak{l}_{2}(A, A), \mathfrak{l}_{2}(A, B)\right)-\mathcal{S}\left(\mathfrak{l}_{1}(B), d B\right)-\mathcal{S}\left(\mathfrak{l}_{1}(B), \mathfrak{l}_{2}(A, B)\right) .
\end{aligned}
$$

By the Jacobi identity that $\mathfrak{l}_{2}$ satisfies and (35), we have

$$
\mathcal{S}\left(\frac{1}{2} \mathfrak{l}_{2}(A, A), \mathfrak{l}_{2}(A, B)\right)=-\mathcal{S}\left(\frac{1}{6} \mathfrak{l}_{2}\left(A, \mathfrak{l}_{2}(A, A)\right), B\right)=0 .
$$

By (35) and the property that $\mathfrak{l}_{2}$ being skew-symmetric, we have

$$
\mathcal{S}\left(\mathfrak{l}_{1}(B), \mathfrak{l}_{2}(A, B)\right)=-\mathcal{S}\left(A, \mathfrak{l}_{2}\left(\mathfrak{l}_{1}(B), B\right)\right)=0 .
$$

It is not hard to see that $d \mathfrak{l}_{1}(B)=\mathfrak{l}_{1} d B$. Thus, by (34), we have

$$
\mathcal{S}\left(\mathfrak{l}_{1}(B), d B\right)=\frac{1}{2} d \mathcal{S}\left(\mathfrak{l}_{1}(B), B\right) .
$$

Finally by (35), we have

$$
\begin{aligned}
d \mathcal{S}\left(\frac{1}{2} \mathfrak{l}_{2}(A, A), B\right) & =\mathcal{S}\left(\frac{1}{2} d \mathfrak{l}_{2}(A, A), B\right)+\mathcal{S}\left(\frac{1}{2} \mathfrak{l}_{2}(A, A), d B\right) \\
& =\mathcal{S}\left(\mathfrak{l}_{2}(d A, A), B\right)+\mathcal{S}\left(\frac{1}{2} \mathfrak{l}_{2}(A, A), d B\right) \\
& =\mathcal{S}\left(d A, \mathfrak{l}_{2}(A, B)\right)+\mathcal{S}\left(\frac{1}{2} \mathfrak{l}_{2}(A, A), d B\right) .
\end{aligned}
$$

Therefore, we have

$$
\mathcal{S}(\text { fcurv }(A, B), \operatorname{curv}(A, B))=d\left(\mathcal{S}(A, d B)+\mathcal{S}\left(\frac{1}{2} \mathfrak{l}_{2}(A, A), B\right)-\frac{1}{2} \mathcal{S}\left(\mathfrak{l}_{1}(B), B\right)\right),
$$

which implies that the first Pontryagin class is trivial. 
At the end of this subsection, we analyze how does the primitive form of the first Pontryagin class behave under the gauge transformation. Recall from [28, Section 5.4] that a gauge transformation between $\Gamma$-connections $(A, B)$ and $\left(A^{\prime}, B^{\prime}\right)$ on $M$ is a pair $(g, \phi)$ consisting of a smooth map $g: M \longrightarrow H_{0}$ and a 1 -form $\phi \in \Omega^{1}\left(M, \mathfrak{h}_{1}\right)$ such that

$$
\begin{aligned}
A^{\prime} & =\operatorname{Ad}_{g} A-g^{*} \bar{\theta}-\mathfrak{l}_{1}(\phi), \\
B^{\prime} & =\left(\Phi_{g}\right)_{*} B-d \phi+\frac{1}{2} \mathfrak{l}_{2}\left(\mathfrak{l}_{1}(\phi), \phi\right)-\mathfrak{l}_{2}\left(\operatorname{Ad}_{\mathfrak{g}} A, \phi\right)+\mathfrak{l}_{2}\left(g^{*} \bar{\theta}, \phi\right),
\end{aligned}
$$

where $\bar{\theta}$ is the Maurer-Cartan 1-form on the Lie group $H_{0}$.

Proposition 6.12. Let $(A, B)$ and $\left(A^{\prime}, B^{\prime}\right)$ be two gauge equivalent $\Gamma$-connections on $M$. Then we have

$$
\mathcal{S}\left(\operatorname{fcurv}\left(A^{\prime}, B^{\prime}\right), \operatorname{curv}\left(A^{\prime}, B^{\prime}\right)\right)=\mathcal{S}(\operatorname{fcurv}(A, B), \operatorname{curv}(A, B)) .
$$

Proof. By the Maurer-Cartan equation that $\bar{\theta}$ satisfies, we have

$$
\begin{aligned}
\operatorname{fcurv}\left(A^{\prime}, B^{\prime}\right)= & d A^{\prime}+\frac{1}{2} \mathfrak{l}_{2}\left(A^{\prime}, A^{\prime}\right)-\mathfrak{l}_{1}\left(B^{\prime}\right) \\
= & d\left(\operatorname{Ad}_{g} A-g^{*} \bar{\theta}-\mathfrak{l}_{1}(\phi)\right)+\frac{1}{2} \mathfrak{l}_{2}\left(\operatorname{Ad}_{g} A-g^{*} \bar{\theta}-\mathfrak{l}_{1}(\phi), \operatorname{Ad}_{g} A-g^{*} \bar{\theta}-\mathfrak{l}_{1}(\phi)\right) \\
& -\mathfrak{l}_{1}\left(\left(\Phi_{g}\right)_{*} B-d \phi+\frac{1}{2} \mathfrak{l}_{2}\left(\mathfrak{l}_{1}(\phi), \phi\right)-\mathfrak{l}_{2}\left(\operatorname{Ad}_{g} A, \phi\right)+\mathfrak{l}_{2}\left(g^{*} \bar{\theta}, \phi\right)\right) \\
= & \operatorname{Ad}_{g} d A+\mathfrak{l}_{2}\left(g^{*} \bar{\theta}, \operatorname{Ad}_{g} A\right)-d g^{*} \bar{\theta}-\mathfrak{l}_{1}(d \phi) \\
& +\frac{1}{2} \operatorname{Ad}_{g} \mathfrak{l}_{2}(A, A)-\mathfrak{l}_{2}\left(\operatorname{Ad}_{g} A, g^{*} \bar{\theta}\right)-\mathfrak{l}_{2}\left(\operatorname{Ad}_{g} A, \mathfrak{l}_{1}(\phi)\right) \\
& +\frac{1}{2} \mathfrak{l}_{2}\left(g^{*} \bar{\theta}, g^{*} \bar{\theta}\right)+\frac{1}{2} \mathfrak{l}_{2}\left(\mathfrak{l}_{1}(\phi), \mathfrak{l}_{1}(\phi)\right)+\mathfrak{l}_{2}\left(g^{*} \bar{\theta}, \mathfrak{l}_{1}(\phi)\right) \\
& -\operatorname{Ad}_{g} \mathfrak{l}_{1}(B)+\mathfrak{l}_{1}(d \phi)-\frac{1}{2} \mathfrak{l}_{2}\left(\mathfrak{l}_{1}(\phi), \mathfrak{l}_{1}(\phi)\right)+\mathfrak{l}_{2}\left(\operatorname{Ad}_{g} A, \mathfrak{l}_{1}(\phi)\right)-\mathfrak{l}_{2}\left(g^{*} \bar{\theta}, \mathfrak{l}_{1}(\phi)\right) \\
= & \operatorname{Ad}_{g}\left(d A+\frac{1}{2} \mathfrak{l}_{2}(A, A)-\mathfrak{l}_{1}(B)\right) \\
= & \operatorname{Ad}_{g} \mathrm{fcurv}(A, B) .
\end{aligned}
$$

Similarly, by a tedious computation, we obtain

$$
\operatorname{curv}\left(A^{\prime}, B^{\prime}\right)=\left(\Phi_{g}\right)_{*} \operatorname{curv}(A, B)-\mathfrak{l}_{2}\left(\operatorname{Ad}_{g} \operatorname{fcurv}(A, B), \phi\right) .
$$

Therefore, by the invariance condition that $\mathcal{S}$ satisfies, we have

$$
\begin{aligned}
\mathcal{S}\left(f \operatorname{fcurv}\left(A^{\prime}, B^{\prime}\right), \operatorname{curv}\left(A^{\prime}, B^{\prime}\right)\right) & =\mathcal{S}\left(\operatorname{Ad}_{g} \operatorname{fcurv}(A, B),\left(\Phi_{g}\right)_{*} \operatorname{curv}(A, B)-\mathfrak{l}_{2}\left(\operatorname{Ad}_{g} f \operatorname{fcurv}(A, B), \phi\right)\right) \\
& =\mathcal{S}(\operatorname{fcurv}(A, B), \operatorname{curv}(A, B))+\mathcal{S}\left(\operatorname{Ad}_{g} \mathfrak{l}_{2}(\operatorname{fcurv}(A, B), \operatorname{fcurv}(A, B)), \phi\right) \\
& =\mathcal{S}(\operatorname{fcurv}(A, B), \operatorname{curv}(A, B)) .
\end{aligned}
$$

The proof is finished.

\section{References}

[1] M. Ammar and N. Poncin, Coalgebraic Approach to the Loday Infinity Category, Stem Differential for $2 n$-ary Graded and Homotopy Algebras. Ann. Inst. Fourier (Grenoble). 60 (1) (2010), 355-387. 
[2] P. Aschieri, L. Cantini and B. Jurčo, Nonabelian bundle gerbes, their differential geometry and gauge theory. Comm. Math. Phys. 254 (2005), no. 2, 367-400.

[3] J. C. Baez and A. S. Crans, Higher-Dimensional Algebra VI: Lie 2-Algebras. Theory Appl. Categ. 12 (2004), 492-528.

[4] J. C. Baez and A. Lauda, Higher-dimensional algebra 5: 2-groups. Theory Appl. Categ. 12 (2004), 423-491.

[5] J. C. Baez and U. Schreiber, Higher gauge theory. Categories in algebra, geometry and mathematical physics, 7-30, Contemp. Math., 431, Amer. Math. Soc., Providence, RI, 2007.

[6] G. Bonavolontà and N. Poncin, On the category of Lie n-algebroids. J. Geom. Phys. 73 (2013), 70-90.

[7] L. Breen and W. Messing, Differential geometry of gerbes. Adv. Math. 198 (2005), no. 2, $732-846$.

[8] P. Bressler, The first Pontryagin class. Compos. Math. 143 (2007), no. 5, 1127-1163.

[9] Z. Chen, M. Stiénon and P. Xu, On regular Courant algebroids. J. Symplectic Geom. 11 (2013), no. 1, 1-24.

[10] F. Girelli and H. Pfeiffer, Higher gauge theory-differential versus integral formulation. J. Math. Phys. 45 (2004), no. 10, 3949-3971.

[11] N. Ikeda and K. Uchino, QP-structures of degree 3 and 4D topological field theory. Comm. Math. Phys. 303 (2011), no. 2, 317-330.

[12] Y. Kosmann-Schwarzbach, Courant algebroids. A short history. SIGMA Symmetry Integrability Geom. Methods Appl. 9 (2013), Paper 014, 8 pp.

[13] J. Liu and Y. Sheng, QP-structures of degree 3 and CLWX 2-algebroids. arXiv:1602.01127.

[14] Z. Liu, A. Weinstein and P. Xu, Manin triples for Lie bialgebroids. J. Diff. Geom. 45(3) (1997), 547-574.

[15] M. Livernet, Homologie des algèbres stables de matrices sur une $A_{\infty}$-algèbre. C. R. Acad. Sci. Paris Sér. I Math. 329(2) (1999), 113-116.

[16] K. C. H. Mackenzie, General theory of Lie groupoids and Lie algebroids. Lecture Note Series, 213. London Mathematical Society. Cambridge University Press, Cambridge, 2005.

[17] J. Martins and A. Miković, Lie crossed modules and gauge-invariant actions for 2-BF theories. Adv. Theor. Math. Phys. 15 (2011), no. 4, 1059-1084.

[18] T. Nikolaus, U. Schreiber and D. Stevenson, Principal $\infty$-bundles: general theory. J. Homotopy Relat. Struct. 10 (2015), no. 4, 749-801.

[19] T. Nikolaus and K. Waldorf, Four equivalent versions of nonabelian gerbes. Pacific J. Math. 264 (2013), no. 2, 355-419.

[20] D. Roytenberg, Courant algebroids, derived brackets and even symplectic supermanifolds. PhD thesis, UC Berkeley, 1999, arXiv:math.DG/9910078. 
[21] D. Roytenberg and A. Weinstein, Courant algebroids and strongly homotopy Lie algebras. Lett. Math. Phys. 46 (1) (1998), 81-93.

[22] U. Schreiber, From Loop Space Mechanics to Nonabelian Strings. Ph. D thesis, University Duisburg-Essen, 2005.

[23] C. Schommer-Pries, Central extensions of smooth 2-groups and a finite-dimensional string 2-group. Geom. Topol. 15 (2011), no. 2, 609-676.

[24] P. Ševera and A. Weinstein, Poisson geometry with a 3-form background. Noncommutative geometry and string theory (Yokohama, 2001). Progr. Theoret. Phys. Suppl. No. 144 (2001), $145-154$

[25] Y. Sheng and Z. Liu, Leibniz 2-algebras and twisted Courant algebroids. Comm. Algebra 41 (2013), no. 5, 1929-1953.

[26] Y. Sheng and C. Zhu, Higher extensions of Lie algebroids. Comm. Contemp. Math. 19 (3) (2017), 1650034, 41 pages.

[27] T. Voronov, Q-manifolds and Higher Analogs of Lie Algebroids. XXIX Workshop on Geometric Methods in Physics. AIP CP 1307, pp. 191-202, Amer. Inst. Phys., Melville, NY, 2010.

[28] K. Waldorf, A global perspective to connections on principal 2-bundles. arXiv:1608.00401,

[29] K. Waldorf, Parallel transport in principle 2-bundle. arXiv:1704.08542.

[30] C. Wockel, Principal 2-bundles and their gauge 2-groups. Forum Math. 23 (2011), no. 3, 565-610. 